\title{
Strong solutions for SPDE with locally monotone coefficients driven by Lévy noise *
}

\author{
Zdzisław Brzeźniak ${ }^{a}$, Wei Liu ${ }^{b, c \dagger}$, Jiahui Zhu ${ }^{d}$ \\ a. Department of Mathematics, University of York, YO105DD York, UK \\ b. School of Mathematical Sciences, Jiangsu Normal University, 221116 Xuzhou, China \\ c. Fakultät für Mathematik, Universität Bielefeld, D-33501 Bielefeld, Germany \\ d. School of Finance, Zhejiang University of Finance and Economics, 310018 Hangzhou, China
}

\begin{abstract}
Motivated by applications to a manifold of semilinear and quasilinear stochastic partial differential equations (SPDEs) we establish the existence and uniqueness of strong solutions to coercive and locally monotone SPDEs driven by Lévy processes. We illustrate the main result of our paper by showing how it can be applied to various types of SPDEs such as stochastic reaction-diffusion equations, stochastic Burgers type equations, stochastic 2D hydrodynamical systems and stochastic equations of nonNewtonian fluids, which generalize many existing results in the literature.
\end{abstract}

AMS Subject Classification: 60H15, 37L30, 34D45

Keywords: Stochastic partial differential equation; Lévy process; local monotonicity; NavierStokes equations; non-Newtonian fluid

\section{Introduction and Main Results}

In recent years, Stochastic Partial Differential Equations (SPDEs) driven by jump type noises such as Lévy-type or Poisson-type perturbations have become extremely popular for modeling financial, physical and biological phenomena. In some circumstances, purely Brownian motion perturbation has many imperfections while capturing some large moves and unpredictable events. Lévy-type perturbations come to the stage to reproduce the performance of those natural phenomena in some real world models. The existence and uniqueness of solutions for SPDEs driven by jump type noises has already been intensively

\footnotetext{
*Supported in part by NSFC (No.11201234), NSF of Jiangsu Higher Education Institutions (No.12KJB110014), the PAPD of Jiangsu Higher Education Institutions, the DFG through SFB-701 and IGK 1132.

${ }^{\dagger}$ Corresponding author: weiliu@math.uni-bielefeld.de
} 
investigated by many authors, see e.g. Kallianpur and Xiong [27], Albeverio et al [2], Mueller et al [43, 44], Applebaum and Wu [3], Mytnik [45], Truman and Wu [58, Hausenblas [25, 26], Mandrekar and Rüdiger [39], Röckner and Zhang [52, Dong et al [16, 17, 18], Marinelli and Röckner [40], Bo et al [5], Brzeźniak et al [9, 10, 12] and the recent monograph by Peszat and Zabczyk [47. The last reference can also be used for more detailed expositions and references.

In this paper, we aim to establish a framework in which one can treat a large number of SPDEs driven by Lévy type noises including stochastic reaction-diffusion equations, stochastic Burgers type equations, stochastic 2D Navier-Stokes equations and stochastic equations of non-Newtonian fluids etc. The line of investigation proposed in this paper began with the celebrated works by Pardoux [46] and Krylov and Rozovskii [29], and later it was further developed by many authors, see e.g. Gyöngy and Krylov [22], Gyöngy [24]. Ren et al [49, Röckner and Wang [51] and Zhang [59]. Roughly speaking, for stochastic equations in finite dimensional spaces, the existence and uniqueness result was obtained under the local monotonicity assumption for the coefficients, see [29] for SDEs driven by Brownian motion and [22] for SDEs driven by (possibly discontinuous) locally square integrable martingales. However, concerning the existence and uniqueness of strong solutions to SPDEs in infinite dimensional spaces driven by Wiener processes or local martingales, all results were established for the globally monotone coefficients SPDE (cf. [29, 24, 49, 59]).

Recently, the classical variational framework has been extended by the second named author and Röckner in [36] for SPDE driven by Wiener process in Hilbert space with locally monotone coefficients. In [36] the authors showed that the local monotonicity method first used by Menaldi and Sritharan [41] for stochastic 2D Navier-Stokes equations (and later used by Sritharan and Sundar [55], Chueshov and Millet [13] for various stochastic equations of hydrodynamics) can be generalized to such an extent that the extended variational framework is applicable to all the equations investigated in [29, 48, 41, 55, 13.

On the other hand, there are not many papers studying non-Lipschitz SPDEs driven by Lévy type noises with small jumps. The first and third named author proved in [1] the existence and uniqueness of solutions to stochastic nonlinear beam equations driven by Lévy type noises. They together with Hausenblas extended in [10] (see also [17]) the work of Menaldi and Sritharan by showing that their method yields the existence and uniqueness of solutions to stochastic 2D Navier Stokes equations driven by a Lévy type noise. There is also the work of the first named author and Hausenblas [9] in which by means of generalized compactness method the existence of solutions to stochastic reaction diffusion equations driven by a Lévy type noise was investigated. What we do in the present paper is to confirm the natural conjecture that the framework in [36] works not only for locally monotone SPDEs driven by multiplicative Gaussian noise but also by multiplicative Lévy type noise (see Remark 1.3). However, we should point out that our results are not applicable to evolution equations with general space time white noise, see for instance [11, 9] and the references therein. The reason is that the solutions of SPDEs with general space time white noise are not regular enough to fit in the variational framework.

The main contribution of this work is that we establish a unified framework for a large class of semilinear and quasilinear SPDE driven by general Lévy noises, which general- 
izes many previous works [46, 29, 24, 36]. The main result is applicable to various types of concrete examples such as stochastic 2D Navier-Stokes equations, stochastic magnetohydrodynamic equations, the Boussinesq model for the Bénard convection, 2D magnetic Bénard problem, stochastic 3D Leray- $\alpha$ model (cf. Remark 4.7) and stochastic equations of non-Newtonian fluids (see Section 4 for details). Hence it also recovers and improves many known results in the literature, see for instance [16, 41, 52, 17, 13, 14, 10]. In a recent work [12 by the first and third named author, a type of stochastic nonlinear beam equations with Poisson-type noises was studied and the existence and uniqueness of solutions was established by following a natural route of constructing a local mild solution and proving, with the help of the Khasminski test, that this solution is a global one. In contrast to [12], the approach used in this paper is different. We will follow the lines in [10, 36] and the technique involves the use of the Galerkin approximation, local monotonicity arguments but not, as opposed to [9], compactness argument. We shall use the result from [22, 1] for the finite dimensional case to construct a sequence of solutions of approximated equations and obtain a prior estimates for those approximated solutions. Then we show that the limit of those approximated solutions solves the original equation by using the local monotonicity arguments.

Now let us describe the framework in more detail. Let

$$
V \subset H \equiv H^{*} \subset V^{*}
$$

be a Gelfand triple, i.e. $\left(H,\langle\cdot, \cdot\rangle_{H}\right)$ is a separable Hilbert space which is identified with its dual space by the Riesz Lemma, $V$ is a reflexive Banach space that is continuously and densely embedded into $H$. If $V^{*}\langle\cdot, \cdot\rangle_{V}$ denotes the duality between $V$ and its dual space $V^{*}$, then we have

$$
V^{*}\langle u, v\rangle_{V}=\langle u, v\rangle_{H}, u \in H, v \in V .
$$

Let $(\Omega, \mathbb{P}, \mathbb{F}, \mathcal{F})$, where $\mathbb{F}=\left(\mathcal{F}_{t}\right)_{t \geq 0}$, is a filtered probability space, $(Z, \mathcal{Z})$ be a measurable space, and $\nu$ be a $\sigma$-finite measure on it. We write

$$
\tilde{N}((0, t] \times B)=N((0, t] \times B)-t \nu(B), t \geq 0, B \in \mathcal{Z}
$$

for the compensated Poisson random measure on $[0, T] \times \Omega \times Z$ associated with a stationary Poisson point process $p$ (see Section 2 for more details). A typical example of $N$ is a Poisson random measure associated with a Lévy process taking values in a separable Banach space. Let $U$ be a separable Hilbert space and let us denote by $\left(\mathcal{T}_{2}(U ; H),\|\cdot\|_{2}\right)$ the Hilbert space of all Hilbert-Schmidt operators from $U$ to $H$. Assume that $\left\{W_{t}\right\}_{t \geq 0}$ is a $U$-valued cylindrical Wiener process on the probability space $(\Omega, \mathbb{P}, \mathbb{F}, \mathcal{F})$. We use the symbol $\mathcal{P}$ to denote the predictable $\sigma$-field, i.e. the $\sigma$-field generated by all left continuous and $\mathbb{F}$-adapted real-valued processes on $[0, T] \times \Omega$. We shall denote by $\mathcal{B F}$ the $\sigma$-field of the progressively measurable sets on $[0, T] \times \Omega$, i.e.

$$
\mathcal{B F}=\left\{A \subset[0, T] \times \Omega: \forall t \in[0, T], A \cap([0, t] \times \Omega) \in \mathcal{B}([0, t]) \otimes \mathcal{F}_{t}\right\}
$$


Now we consider a type of SPDEs driven by Lévy processes of the following form:

$$
\begin{aligned}
\mathrm{d} X_{t}= & A\left(t, X_{t}\right) \mathrm{d} t+B\left(t, X_{t}\right) \mathrm{d} W_{t} \\
& +\int_{D^{c}} f\left(t, X_{t-}, z\right) \tilde{N}(\mathrm{~d} t, \mathrm{~d} z)+\int_{D} g\left(t, X_{t-}, z\right) N(\mathrm{~d} t, \mathrm{~d} z), \\
X_{0}= & x,
\end{aligned}
$$

where $x$ is an $\mathcal{F}_{0}$-measurable random variable, $A:[0, T] \times \Omega \times V \rightarrow V^{*}$ and $B:[0, T] \times \Omega \times V \rightarrow$ $\mathcal{T}_{2}(U ; H)$ are both $\mathcal{B F} \otimes \mathcal{B}(V)$-measurable functions, $D \in \mathcal{Z}$ with $\mathbb{E} N((0, t] \times D)<\infty$ for every $0<t \leq T$, and $f, g:[0, T] \times \Omega \times V \times Z \rightarrow H$ are $\mathcal{P} \otimes \mathcal{B}(V) \otimes \mathcal{Z}$-measurable functions.

The main aim of this work is to establish the existence and uniqueness of strong solutions to (1.1) under the coercivity and local monotonicity conditions.

For this purpose, let us first formulate the main assumptions on the coefficients.

Suppose that there exist constants $\alpha>1, \beta \geq 0, \theta>0, C>0$, a positive $\mathbb{F}$-adapted process $F$ and a measurable, bounded on balls function $\rho: V \rightarrow[0,+\infty)$ such that the following conditions hold for all $v, v_{1}, v_{2} \in V$ and $(t, \omega) \in[0, T] \times \Omega$ :

(H1) (Hemicontinuity) The map $s \mapsto V^{*}\left\langle A\left(t, v_{1}+s v_{2}\right), v\right\rangle_{V}$ is continuous on $\mathbb{R}$.

(H2) (Local monotonicity)

$$
\begin{aligned}
& 2_{V^{*}}\left\langle A\left(t, v_{1}\right)-A\left(t, v_{2}\right), v_{1}-v_{2}\right\rangle_{V}+\left\|B\left(t, v_{1}\right)-B\left(t, v_{2}\right)\right\|_{2}^{2} \\
+ & \int_{D^{c}}\left\|f\left(t, v_{1}, z\right)-f\left(t, v_{2}, z\right)\right\|_{H}^{2} \nu(\mathrm{d} z) \leq\left(C+\rho\left(v_{2}\right)\right)\left\|v_{1}-v_{2}\right\|_{H}^{2},
\end{aligned}
$$

(H3) (Coercivity)

$$
2_{V^{*}}\langle A(t, v), v\rangle_{V}+\|B(t, v)\|_{2}^{2}+\theta\|v\|_{V}^{\alpha} \leq F_{t}+C\|v\|_{H}^{2}
$$

(H4) (Growth)

$$
\|A(t, v)\|_{V^{*}}^{\frac{\alpha}{\alpha-1}} \leq\left(F_{t}+C\|v\|_{V}^{\alpha}\right)\left(1+\|v\|_{H}^{\beta}\right)
$$

Definition 1.1. (Solution of SEE) An $H$-valued càdlàg $\mathbb{F}$-adapted process $\left\{X_{t}\right\}_{t \in[0, T]}$ is called a solution of (1.1) , if for its $\mathrm{d} t \times \mathbb{P}$-equivalent class $\bar{X}$ we have

(1) $\bar{X} \in L^{\alpha}([0, T] ; V) \cap L^{2}([0, T] ; H), \mathbb{P}$-a.s.;

(2) the following equality holds $\mathbb{P}$-a.s.:

$$
\begin{aligned}
X_{t}=x & +\int_{0}^{t} A\left(s, \bar{X}_{s}\right) \mathrm{d} s+\int_{0}^{t} B\left(s, \bar{X}_{s}\right) \mathrm{d} W_{s} \\
& +\int_{0}^{t} \int_{D^{c}} f\left(s, \bar{X}_{s-}, z\right) \tilde{N}(\mathrm{~d} s, \mathrm{~d} z)+\int_{0}^{t} \int_{D} g\left(s, \bar{X}_{s-}, z\right) N(\mathrm{~d} s, \mathrm{~d} z), t \in[0, T] .
\end{aligned}
$$


Remark 1.1. The integrability of all terms in the above equality are implicitly required in the definition and it will be all justified in the proof of existence of solutions. Note that $A\left(s, \bar{X}_{s}\right)$ is a $V^{*}$-valued process according to the definition, however, the integral with respect to $\mathrm{d} s$ in the above equality is initially a $V^{*}$-valued Bochner integral which turns out to be in fact $H$-valued.

Now we can present the main result of this paper.

Theorem 1.2. Suppose that conditions $(H 1)-(H 4)$ hold for $F \in L^{\frac{\beta+2}{2}}([0, T] \times \Omega ; \mathrm{d} t \times \mathbb{P})$, and there exists constant $\gamma<\frac{\theta}{2 \beta}$ such that for all $t \in[0, T], \omega \in \Omega$ and $v \in V$ we have

$$
\begin{aligned}
& \|B(t, v)\|_{2}^{2}+\int_{D^{c}}\|f(t, v, z)\|_{H}^{2} \nu(\mathrm{d} z) \leq F_{t}+C\|v\|_{H}^{2}+\gamma\|v\|_{V}^{\alpha} ; \\
& \int_{D^{c}}\|f(t, v, z)\|_{H}^{\beta+2} \nu(\mathrm{d} z) \leq F_{t}^{\frac{\beta+2}{2}}+C\|v\|_{H}^{\beta+2} ; \\
& \rho(v) \leq C\left(1+\|v\|_{V}^{\alpha}\right)\left(1+\|v\|_{H}^{\beta}\right) .
\end{aligned}
$$

(i) Then for any $x \in L^{\beta+2}\left(\Omega, \mathcal{F}_{0}, \mathbb{P} ; H\right)$, Equation (1.1) has a unique solution $\left\{X_{t}\right\}_{t \in[0, T]}$.

(ii) If $g \equiv 0$, then there exists a constant $C$ such that

$$
\sup _{t \in[0, T]} \mathbb{E}\left\|X_{t}\right\|_{H}^{\beta+2}+\mathbb{E} \int_{0}^{T}\left\|X_{t}\right\|_{H}^{\beta}\left\|X_{t}\right\|_{V}^{\alpha} \mathrm{d} t \leq C\left(\mathbb{E}\|x\|_{H}^{\beta+2}+\mathbb{E} \int_{0}^{T} F_{t}^{(\beta+2) / 2} \mathrm{~d} t\right) .
$$

(iii) If $g \equiv 0$ and $\gamma$ is small enough, then we have

$$
\mathbb{E}\left(\sup _{t \in[0, T]}\left\|X_{t}\right\|_{H}^{\beta+2}\right)+\mathbb{E} \int_{0}^{T}\left\|X_{t}\right\|_{H}^{\beta}\left\|X_{t}\right\|_{V}^{\alpha} \mathrm{d} t \leq C\left(\mathbb{E}\|x\|_{H}^{\beta+2}+\mathbb{E} \int_{0}^{T} F_{t}^{(\beta+2) / 2} \mathrm{~d} t\right) .
$$

Remark 1.3. (1) If $f=g \equiv 0$ in (1.1) (i.e. Wiener noise case), then Theorem 1.2 recovers the main result in [36]. Moreover, we improve [36, Theorem 1.1] for allowing a positive constant $\gamma$ in (1.2), which means that the diffusion coefficient $B$ can also depend on some gradient term of the solution in applications. We also want to emphasize that $(H 2)$ is essentially weaker than the classical monotonicity condition used extensively in the literature (i.e. $\rho \equiv 0$, see e.g. [46, 29, 48, 52, 49, 21]). The typical examples are the stochastic Burgers equations and 2D Navier-Stokes equation (see Remark 4.7 for many other examples) on a bounded or unbounded domain, which satisfies (H2) but does not satisfy the standard monotonicity condition (cf. Section 4 for the details).

(2) If $g \equiv 0$ in (1.1), $\rho \equiv 0, \alpha=2, \beta=0$ in $(H 2)$-( $H 4)$, then the existence and uniqueness of strong solutions to (1.1). follows from the general result of Gyöngy [24].

(3) If the noise is zero or additive type in (1.1), then the (local) existence and uniqueness of solutions is established in [35, 37] by replacing $(H 2)$ with the following more general local monotonicity condition:

$$
V^{*}\left\langle A\left(t, v_{1}\right)-A\left(t, v_{2}\right), v_{1}-v_{2}\right\rangle_{V} \leq\left(K+\eta\left(v_{1}\right)+\rho\left(v_{2}\right)\right)\left\|v_{1}-v_{2}\right\|_{H}^{2},
$$


where $\eta, \rho: V \rightarrow[0,+\infty)$ are measurable functions and locally bounded in $V$.

(4) In general, the estimates (1.5) and (1.6) might not hold anymore if we have large jumps term in the equation. However, if we assume that the Lévy measure has finite moment of certain order (see e.g.[18]), then it is still possible to obtain some similar estimates. This subject and some related applications will be investigated in future works.

Remark 1.4. (1) Note that if $\beta=0$ in (H4), then one can just take any $\gamma<\infty$ in (1.2). In this case, the assumption on $B$ in (1.2) can be removed since it follows directly from $(H 3)$ and $(H 4)$ (cf. [48, Remark 4.1.1]).

(2) If $f$ satisfies the following growth condition for some fixed $p \geq \beta+2$ :

$$
\|f(t, v, z)\|_{H}^{p} \leq h(z)^{p}\left(F_{t}^{\frac{p}{2}}+C\|v\|_{H}^{p}\right), \quad(t, v, z) \in[0, T] \times V \times D^{c},
$$

where $\int_{D^{c}}\left[h(z)^{\beta+2}+h(z)^{2}\right] \nu(d z)<\infty$, then it is easy to show that conditions (1.3) and (1.2) hold.

In particular, if $f$ satisfying the following conditions:

$$
\begin{aligned}
& \|f(t, x, z)-f(t, y, z)\|_{H} \leq C\|x-y\|_{H}\|z\|, t \in[0, T], x, y \in V, z \in D^{c} \\
& \|f(t, x, z)\|_{H} \leq C\left(1+\|x\|_{H}\right)\|z\|, t \in[0, T], x \in V, z \in D^{c},
\end{aligned}
$$

where $\int_{D^{c}}\|z\|^{2} \nu(\mathrm{d} z)<\infty$, then $(H 2)$, (1.2) and (1.3) are all fulfilled.

The rest of the paper is organized as follows: in the next section we will recall some preliminaries on the Poisson random measure and its corresponding stochastic integral. The proof of the main result will be given in Section 3 and some concrete examples of SPDE will be studied in Section 4 as applications. Note that we always use $C$ to denote a generic constant which may change from line to line.

\section{Some Preliminaries on Poisson Random Measure}

We begin with a brief review of terminology and results on Poisson random measures. Let $(S, \mathcal{S})$ be a measurable space, $\mathbb{N}=\{0,1,2, \cdots\}$ and $\overline{\mathbb{N}}=\mathbb{N} \cup\{\infty\}$. Let $\mathbb{M}_{\overline{\mathbb{N}}}(S)$ denote the space of all $\overline{\mathbb{N}}$-valued measures on $(S, \mathcal{S})$. We use the symbol $\mathcal{B}\left(\mathbb{M}_{\overline{\mathbb{N}}}(S)\right)$ to denote the smallest $\sigma$-field on $\mathbb{M}_{\overline{\mathbb{N}}}(S)$ with respect to which all mappings $i_{B}: \mathbb{M}_{\overline{\mathbb{N}}}(S) \ni \mu \mapsto \mu(B) \in \overline{\mathbb{N}}$, $B \in \mathcal{S}$ are measurable.

Definition 2.1. A map $N: \Omega \times \mathcal{S} \rightarrow \overline{\mathbb{N}}$ is called an $\overline{\mathbb{N}}$-valued random measure if for each $\omega \in \Omega, N(\omega, \cdot) \in \mathbb{M}_{\overline{\mathbb{N}}}(S)$ and for each $A \in \mathcal{S}, N(\cdot, A)$ is an $\overline{\mathbb{N}}$-valued random variable on the probability space $(\Omega, \mathbb{P}, \mathcal{F})$. We will often write $N(A)$ instead of $N(\cdot, A)$ for simplicity of notation.

Definition 2.2. An $\overline{\mathbb{N}}$-valued random measure $N$ is called a Poisson random measure if

(1) for any $B \in \mathcal{S}$ satisfying $\mathbb{E}[N(B)]<\infty, N(B)$ is a Poisson random variable with parameter $\eta(B)=\mathbb{E}[N(B)]$; 
(2) for any pairwise disjoint sets $B_{1}, \cdots, B_{n} \in \mathcal{S}$, the random variables

$$
N\left(B_{1}\right), \cdots, N\left(B_{n}\right)
$$

are independent.

Let $(Z, \mathcal{Z})$ be a measurable space. A point function $\alpha$ on $(Z, \mathcal{Z})$ is a mapping $\alpha$ : $\mathcal{D}(\alpha) \rightarrow Z$, where the domain $\mathcal{D}(\alpha)$ of $\alpha$ is a countable subset of $(0, \infty)$. Let $\Pi_{Z}$ be the set of all point functions on $Z$. For each point function, we define a counting measure $N_{\alpha}$ by

$$
N_{\alpha}(U):=\sharp\{s \in \mathcal{D}(\alpha):(s, \alpha(s)) \in U\}, \quad U \in \mathcal{B}((0, \infty)) \otimes \mathcal{Z} .
$$

Denote by $\mathcal{Q}$ the $\sigma$-field on $\Pi_{Z}$ generated by all the subsets $\left\{\alpha \in \Pi_{Z}: N_{\alpha}(U)=k\right\}$, $U \in \mathcal{Z}, k=0,1,2, \cdots$. A function $p: \Omega \rightarrow \Pi_{Z}$ is called a point process on $Z$ if it is $\mathcal{F} / \mathcal{Q}$-measurable. Let $p$ be a point process on $Z$. We can define the counting measure $N_{p}$ associated with $p$ by

$$
N_{p}(U, \omega):=\sharp\{s \in \mathcal{D}(p(\omega)):(s, p(s, \omega)) \in U\}, \quad U \in \mathcal{B}((0, \infty)) \otimes \mathcal{Z}, \omega \in \Omega .
$$

In particular, we have

$$
N_{p}((0, t] \times A, \omega)=\sharp\{s \in(0, t] \cap \mathcal{D}(p(\omega)): p(s, \omega) \in A\}, \quad A \in \mathcal{Z}, \quad 0<t \leq T .
$$

It is also useful to introduce the shifted point process $\theta_{t} p, t \geq 0$ defined by

$$
\begin{aligned}
\left(\theta_{t} p\right)(s) & =p(s+t), s>0 \\
\mathcal{D}\left(\theta_{t} p\right) & =\{s \in(0, \infty): s+t \in \mathcal{D}(p)\} .
\end{aligned}
$$

and the stopped point process $\alpha_{t} p$ defined by

$$
\begin{aligned}
\left(\alpha_{t} p\right)(s) & =p(s), \text { for } s \in \mathcal{D}\left(\alpha_{t} p\right) ; \\
\mathcal{D}\left(\alpha_{t} p\right) & =(0, t] \cap \mathcal{D}(p) .
\end{aligned}
$$

Definition 2.3. A point process $p$ is said to be finite if $\mathbb{E} N_{p}((0, t] \times Z)<\infty$ for every $0<t \leq T$.

A point process $p$ is said to be $\sigma$-finite if there exists an increasing sequence $\left\{D_{n}\right\}_{n \in \mathbb{N}} \subset \mathcal{Z}$ such that $\cup_{n} D_{n}=Z$ and $\mathbb{E} N_{p}\left((0, t] \times D_{n}\right)<\infty$ for all $0<t \leq T$ and $n \in \mathbb{N}$.

A point process $p$ is said to be stationary if for every $t>0, p$ and $\theta_{t} p$ have the same probability laws.

A point process $p$ is said to be renewal if it is stationary and for every $0<t<\infty$, the point processes $\alpha_{t} p$ and $\theta_{t} p$ are independent.

A point process $p$ is said to be adapted to the filtration $\mathbb{F}$ if for every $t>0$ and $A \in \mathcal{Z}$, its counting measure $N_{p}((0, t] \times A)$ is $\mathcal{F}_{t}$-measurable.

A point process $p$ is called a Poisson point process if $N_{p}(\cdot)$ defined by (2.1) is a Poisson random measure on $((0, \infty) \times Z, \mathcal{B}((0, \infty)) \otimes \mathcal{Z})$. 
Remark 2.1. It can be shown that if a point process $p$ is $\sigma$-finite and renewal, then $N_{p}$ defined by (2.1) is a Poisson random measure (cf. [33, Theorem 3.1]). It is easy to verify that a Poisson point process is stationary if and only if there exists a nonnegative measure $\nu$ on $(Z, \mathcal{Z})$ such that

$$
\mathbb{E} N_{p}((0, t] \times A)=t \nu(A), \quad t>0, \quad A \in \mathcal{Z} .
$$

In such a case, we say that the Poisson random measure $N_{p}$ is time homogenous. At this point, it should be mentioned that, in the literature, some authors may use the above property (2.3) as an alternative definition of stationary property of a Poisson point process. In fact, this is consistent with our definition of a stationary point process.

Let $\mathcal{M}_{T}^{q}(\mathcal{P} \otimes \mathcal{Z}, \mathrm{d} t \times \mathbb{P} \times \nu ; H), q \in[1, \infty)$, be the space of all (equivalence classes of) $\mathcal{P} \otimes \mathcal{Z}$-measurable functions $f:[0, T] \times \Omega \times Z \rightarrow H$ such that

$$
\mathbb{E} \int_{0}^{T} \int_{Z}\|f(s, \cdot, z)\|_{H}^{q} \nu(\mathrm{d} z) \mathrm{d} s<\infty .
$$

Let $\mathcal{M}_{T}(\mathcal{P} \otimes \mathcal{Z}, N ; H)$ be the space of all $\mathcal{P} \otimes \mathcal{Z}$-measurable functions $f:[0, T] \times \Omega \times Z \rightarrow H$ such that

$$
\mathbb{E} \int_{0}^{T} \int_{Z}\|f(s, \cdot, z)\|_{H} N(\mathrm{~d} s, \mathrm{~d} z)<\infty .
$$

Here $\int_{0}^{T} \int_{Z}\|f(s, \omega, z)\|_{H} N(\mathrm{~d} s, \mathrm{~d} z)(\omega)$ is understood to be the Lebesgue integral w.r.t. the measure $N(\cdot, \cdot)(\omega)$ for every $\omega \in \Omega$ and is equal to the convergent sum (cf. [31]),

$$
\int_{0}^{T} \int_{Z}\|f(s, \omega, z)\|_{H} N(\mathrm{~d} s, \mathrm{~d} z)(\omega)=\sum_{s \in(0, T] \cap \mathcal{D}(p(\omega))}\|f(s, \omega, p(s, \omega))\|_{H} .
$$

It should come as no surprise that if $f:[0, T] \times \Omega \times Z \rightarrow H$ is a $\mathcal{B}([0, T]) \otimes \mathcal{F}_{T} \otimes \mathcal{Z}$ measurable function and $\mathbb{E} \int_{0}^{T} \int_{Z}\|f(s, \cdot, z)\|_{H} N(d s, d z)<\infty$, then for every $\omega \in \Omega, f(\cdot, \omega, \cdot)$ is $\mathcal{B}([0, T]) \otimes \mathcal{Z}$-measurable and $\int_{0}^{T} \int_{Z}\|f(s, \omega, z)\|_{H} N(\mathrm{~d} s, \mathrm{~d} z)(\omega)<\infty$, $\mathbb{P}$-a.s.. Hence for almost all $\omega \in \Omega, f(\cdot, \omega, \cdot)$ is Bochner integrable with respect to $N(\mathrm{~d} s, \mathrm{~d} z)(\omega)$ and we have for every $t \leq T$

$$
\int_{0}^{t} \int_{Z} f(s, \omega, z) N(\mathrm{~d} s, \mathrm{~d} z)(\omega)=\sum_{s \in(0, t] \cap \mathcal{D}(p(\omega))} f(s, \omega, p(s, \omega)), \mathbb{P} \text {-a.s. }
$$

Now we state some important properties of the stochastic integrals w.r.t. the compensated Poisson random measures, where the proofs of these properties and more detailed discussions can be found in [31] (see also [8, 53, 61]).

Proposition 2.2. Assume $f \in \mathcal{M}_{T}^{2}(\mathcal{P} \otimes \mathcal{Z}, \mathrm{d} t \times \mathbb{P} \times \nu ; H)$. Then the following conclusions hold: 
(i) The stochastic integral process $\int_{0}^{t} \int_{Z} f(s, \cdot, z) \tilde{N}(\mathrm{~d} s, \mathrm{~d} z), t \in[0, T]$ is a càdlàg 2-integrable martingale. More precisely, it has a modification which has càdlàg trajectories.

(ii) The isometry property:

$$
\mathbb{E}\left\|\int_{0}^{t} \int_{Z} f(s, \cdot, z) \tilde{N}(\mathrm{~d} s, \mathrm{~d} z)\right\|_{H}^{2}=\mathbb{E} \int_{0}^{t} \int_{Z}\|f(s, \cdot, z)\|_{H}^{2} \nu(\mathrm{d} z) \mathrm{d} s, \quad t \in(0, T] .
$$

(iii) If $D \in \mathcal{Z}$ with $\mathbb{E}(N((0, t] \times D))<\infty$, then for every $t \in[0, T], \mathbb{P}$-a.s.,

$$
\int_{0}^{t} \int_{D} f(s, \cdot, z) \tilde{N}(\mathrm{~d} s, \mathrm{~d} z)=\sum_{s \in(0, t] \cap \mathcal{D}(p)} f(s, \cdot, p(s)) 1_{D}(p(s))-\int_{0}^{t} \int_{D} f(s, \cdot, z) \nu(\mathrm{d} z) \mathrm{d} s ;
$$

(iv) If in addition $f \in \mathcal{M}_{T}^{1}(\mathcal{P} \otimes \mathcal{Z}, \mathrm{d} t \times \mathbb{P} \times \nu ; H)$, then for each $t \in[0, T]$, $\mathbb{P}$-a.s.

$$
\int_{0}^{t} \int_{Z} f(s, \cdot, z) \tilde{N}(\mathrm{~d} s, \mathrm{~d} z)=\sum_{s \in(0, t] \cap \mathcal{D}(p)} f(s, \cdot, p(s))-\int_{0}^{t} \int_{Z} f(s, \cdot, z) \nu(\mathrm{d} z) \mathrm{d} s .
$$

Remark 2.3. (1) We may extend the stochastic integral to $\mathcal{P} \otimes \mathcal{Z}$-measurable functions $f$ satisfying

$$
\int_{0}^{T} \int_{Z}\|f(s, \cdot, z)\|_{H}^{2} \nu(\mathrm{d} z) \mathrm{d} s<\infty, \mathbb{P} \text {-a.s. }
$$

In this case, the process $\int_{0}^{t} \int_{Z} f(s, \cdot, z) \tilde{N}(\mathrm{~d} s, \mathrm{~d} z), t \in[0, T]$ is a càdlàg 2-integrable local martingale and for every stopping time $\tau \leq T$, we have

$$
\int_{0}^{t \wedge \tau} \int_{Z} f(s, \cdot, z) \tilde{N}(\mathrm{~d} s, \mathrm{~d} z)=\int_{0}^{t} \int_{Z} 1_{[0, \tau]} f(s, \cdot, z) \tilde{N}(\mathrm{~d} s, \mathrm{~d} z) .
$$

(2) From now on, whenever we use the stochastic process $\int_{0}^{t} \int_{Z} f(s, \cdot, z) \tilde{N}(\mathrm{~d} s, \mathrm{~d} z), t \in$ $[0, T]$, we implicitly assume that it has càdlàg trajectories, in which case, the stochastically equivalence coincides with the $\mathbb{P}$-equivalence.

(3) For Banach spaces martingale type $p(1<p \leq 2)$, one has, instead of the Itô isometry property (2.7), the following continuity property $\left(C_{p}\right.$ is some constant)(cf. [61]):

$$
\mathbb{E}\left\|\int_{0}^{T} \int_{Z} f(s, \cdot, z) \tilde{N}(\mathrm{~d} s, \mathrm{~d} z)\right\|_{H}^{p} \leq C_{p} \mathbb{E} \int_{0}^{T} \int_{Z}\|f(s, \cdot, z)\|_{H}^{p} \nu(\mathrm{d} z) \mathrm{d} s .
$$

(4) Even though there are close connections between predictable processes and progressively measurable processes, the predictability requirement of the function $f$ in Proposition 2.2 ( $i i i)$ and $(i v)$ is necessary. In fact, one can find a progressively measurable but not predictable function such that identities (2.8) and (2.9) no longer hold (cf. [61]). 
One should note that another important and widely used class of Poisson random measures are the one associated to a Lévy process, which is actually a special type of Poisson random measures associated to a Poisson point process as we discussed before. More precisely, let $L:=\left(L_{t}\right)_{t \geq 0}$ be an $Z$-valued Lévy process, where $Z$ is a separable Banach space. Without loss of generality we may assume that the Lévy process $L$ is càdlàg, even if we don't impose the càdlàg property in the definition of a Lévy process, see e.g. [15, Theorem 16.1]. Hence, for every $\omega \in \Omega, L$. $(\omega)$ has at most countable number of jumps on $[0, t]$. So it is easy to see that for every $\omega \in \Omega$,

$$
\triangle L .(\omega):[0, \infty) \rightarrow Z ; \triangle L_{s}(\omega):=L_{s}(\omega)-L_{s-}(\omega)
$$

is a point function in $(Z \backslash\{0\}, \mathcal{B}(Z \backslash\{0\}))$. Let us define a function $N$ by

$$
N(A, \omega)=\sharp\left\{s \in(0, \infty):\left(s, \triangle L_{s}(\omega)\right) \in A\right\}, \quad A \in \mathcal{B}((0, \infty)) \otimes \mathcal{B}(Z \backslash\{0\}), \omega \in \Omega .
$$

It is easy to check that $\triangle L: \Omega \rightarrow \Pi_{Z}$ is $\mathcal{F} / \mathcal{Q}$-measurable. Thereby $\triangle L$ is a point process. Since the Lévy process $L$ has independent and stationary increments, one can show that the point process $\triangle L$ is stationary and renewal. Obviously, by taking $D_{n}=\left\{x \in Z:\|x\|>\frac{1}{n}\right\}$, we find that the point process $\triangle L$ is $\sigma$-finite. On the basis of Remark 2.1, we know that the function $N$ defined by equality $(2.10)$ is a stationary Poisson random measure

$$
\mathbb{E} N((0, t] \times A)=t \nu(A), t>0, A \in \mathcal{B}(Z \backslash\{0\}),
$$

where $\nu$ is a nonnegative measure. In this case, $N$ is called the Poisson random measure associated to the Lévy process $L$.

\section{Proof of The Theorem 1.2}

\subsection{The case without large jumps}

First of all we note that since $\nu(D)<\infty$, the set

$$
\{s \in(0, T] \cap \mathcal{D}(p): p(s, \omega) \in D\}
$$

contains only finitely many points for almost all $\omega \in \Omega$. Put

$$
\begin{aligned}
\tau_{1} & =\inf \{s \in(0, \infty) \cap \mathcal{D}(p): p(s) \in D\} \wedge T \\
\tau_{m} & =\inf \left\{s \in(0, \infty) \cap \mathcal{D}(p): p(s) \in D ; s>\tau_{m-1}\right\} \wedge T, m \geq 2 .
\end{aligned}
$$

The random times $\tau_{1}, \tau_{2}, \cdots$ form a random configuration of points in $(0, T]$ with $p\left(\tau_{i}\right) \in D$ and it is a sequence of jump times of the Poisson process $N(t, D):=N((0, t] \times D), t \in(0, T]$. We see at once that $\tau_{m} \uparrow T$ as $m \rightarrow \infty \mathbb{P}$-a.s. and for each $m$, the random time $\tau_{m}$ is a stopping time. Note that since $\int_{0}^{t} g\left(s, X_{s-}, z\right) N(\mathrm{~d} s, \mathrm{~d} z)=0$ for $t \in\left[0, \tau_{1}\right)$, the equation (1.1) on the interval $\left[0, \tau_{1}\right)$ can be rewritten into the following type of equation:

$$
\begin{aligned}
& \mathrm{d} X_{t}=A\left(t, X_{t}\right) \mathrm{d} t+B\left(t, X_{t}\right) \mathrm{d} W_{t}+\int_{D^{c}} f\left(t, X_{t-}, z\right) \tilde{N}(\mathrm{~d} t, \mathrm{~d} z), t \in\left[0, \tau_{1}\right), \\
& X_{0}=x .
\end{aligned}
$$


Actually, by means of the interlacing procedure (which will be introduced in Section 3.2 and cf. also Theorem 9.1 in [31]), for the proof of Theorem 1.2 it is sufficient to show the existence and uniqueness of solutions to (3.1).

Theorem 3.1. Under the assumptions of Theorem 1.2 , for every $x \in L^{\beta+2}\left(\Omega, \mathcal{F}_{0}, \mathbb{P} ; H\right)$, there exists a unique càdlàg $H$-valued $\mathbb{F}$-adapted process $\left(X_{t}\right)$ such that $\mathbb{P}$-a.s.:

$$
X_{t}=x+\int_{0}^{t} A\left(s, \bar{X}_{s}\right) \mathrm{d} s+\int_{0}^{t} B\left(s, \bar{X}_{s}\right) \mathrm{d} W_{s}+\int_{0}^{t} \int_{D^{c}} f\left(s, \bar{X}_{s-}, z\right) \tilde{N}(\mathrm{~d} s, \mathrm{~d} z), t \in[0, T],
$$

where $\bar{X} \in L^{\alpha}([0, T] \times \Omega, \mathrm{d} t \times \mathbb{P} ; V) \cap L^{2}([0, T] \times \Omega, \mathrm{d} t \times \mathbb{P} ; H)$ and it is $\mathrm{d} t \times \mathbb{P}$-equivalent to $X$.

Moreover, we have

$$
\sup _{t \in[0, T]} \mathbb{E}\left\|X_{t}\right\|_{H}^{\beta+2}+\mathbb{E} \int_{0}^{T}\left\|X_{t}\right\|_{H}^{\beta}\left\|X_{t}\right\|_{V}^{\alpha} \mathrm{d} t \leq C\left(\mathbb{E}\|x\|_{H}^{\beta+2}+\mathbb{E} \int_{0}^{T} F_{t}^{(\beta+2) / 2} \mathrm{~d} t\right) .
$$

The proof of Theorem 3.1 is divided into three steps. Assume that $\left\{e_{1}, e_{2}, \cdots\right\} \subset V$ is an orthonormal basis of $H$ such that $\operatorname{span}\left\{e_{1}, e_{2}, \cdots\right\}$ is dense in $V$. Denote $H_{n}:=$ $\operatorname{span}\left\{e_{1}, \cdots, e_{n}\right\}$. Let $P_{n}: V^{*} \rightarrow H_{n}$ be defined by

$$
P_{n} y:=\sum_{i=1}^{n} V^{*}\left\langle y, e_{i}\right\rangle_{V} e_{i}, y \in V^{*}
$$

It is easy to see that $\left.P_{n}\right|_{H}$ is just the orthogonal projection onto $H_{n}$ in $H$ and we have

$$
V^{*}\left\langle P_{n} A(t, u), v\right\rangle_{V}=\left\langle P_{n} A(t, u), v\right\rangle_{H}=V^{*}\langle A(t, u), v\rangle_{V}, u \in V, v \in H_{n} .
$$

Let $\left\{g_{1}, g_{2}, \cdots\right\}$ be an orthonormal basis of $U$ and

$$
W_{t}^{(n)}:=\sum_{i=1}^{n}\left\langle W_{t}, g_{i}\right\rangle_{U} g_{i}=\tilde{P}_{n} W_{t}
$$

where $\tilde{P}_{n}$ is the orthogonal projection from $U$ onto $\operatorname{span}\left\{g_{1}, \cdots, g_{n}\right\}$.

For each $n \in \mathbb{N}$, we consider the following stochastic differential equation on $H_{n}$ :

$$
\begin{aligned}
\mathrm{d} X_{t}^{(n)} & =P_{n} A\left(t, X_{t}^{(n)}\right) \mathrm{d} t+P_{n} B\left(t, X_{t}^{(n)}\right) \mathrm{d} W_{t}^{(n)}+\int_{D^{c}} P_{n} f\left(t, X_{t-}^{(n)}, z\right) \tilde{N}(\mathrm{~d} t, \mathrm{~d} z), \\
X_{0}^{(n)} & =P_{n} x .
\end{aligned}
$$

According to [22, Theorem 1] (cf. also [1, Theorem 3.1]), (3.4) has a unique strong solution, i.e. satisfying the following integral equation:

$$
\begin{aligned}
X_{t}^{(n)}= & P_{n} x+\int_{0}^{t} P_{n} A\left(s, X_{s}^{(n)}\right) \mathrm{d} t+\int_{0}^{t} P_{n} B\left(s, X_{s}^{(n)}\right) \mathrm{d} W_{s}^{(n)} \\
& +\int_{0}^{t} \int_{D^{c}} P_{n} f\left(s, X_{s-}^{(n)}, z\right) \tilde{N}(\mathrm{~d} s, \mathrm{~d} z), \quad t \in[0, T] .
\end{aligned}
$$



$X^{(n)}$.

In order to construct the solution to equation (3.1), we need to find a priori estimate for

Lemma 3.2. Under the assumptions of Theorem 1.2, there exists $C>0$ such that

$$
\begin{aligned}
& \sup _{n \in \mathbb{N}}\left(\sup _{t \in[0, T]} \mathbb{E}\left\|X_{t}^{(n)}\right\|_{H}^{\beta+2}+\mathbb{E} \int_{0}^{T}\left\|X_{t}^{(n)}\right\|_{H}^{\beta}\left\|X_{t}^{(n)}\right\|_{V}^{\alpha} \mathrm{d} t\right) \\
\leq & C\left(\mathbb{E}\|x\|_{H}^{\beta+2}+\mathbb{E} \int_{0}^{T} F_{t}^{(\beta+2) / 2} \mathrm{~d} t\right) .
\end{aligned}
$$

Proof. For any given $n \in \mathbb{N}$, we define

$$
\tau_{R}^{(n)}:=\inf \left\{t \geq 0:\left\|X_{t}^{(n)}\right\|_{H}>R\right\} \wedge T .
$$

Since the solution $\left(X_{t}^{(n)}\right)_{0 \leq t \leq T}$ is right continuous and $\mathbb{F}$-adapted, $\tau_{R}^{(n)}$ is a stopping time for every $R \in \mathbb{N}$. Moreover, since the trajectories of $X^{(n)}$ are $\mathbb{P}$-a.s. right continuous with left limits, the process $X^{(n)}$ is bounded on every compact intervals. Hence we see that $\tau_{R}^{(n)} \uparrow T$ , $\mathbb{P}$-a.s. and $\mathbb{P}\left\{\tau_{R}^{(n)}<T\right\}=0$ as $R \rightarrow \infty$.

For the simplicity of notations we take $p=\beta+2$. By applying the Itô formula (cf. [42]) to the function $\|\cdot\|_{H}^{p}$ and the process $X_{t}^{(n)}$ we have

$$
\begin{aligned}
\left\|X_{t}^{(n)}\right\|_{H}^{p}= & \left\|X_{0}^{(n)}\right\|_{H}^{p}+p(p-2) \int_{0}^{t}\left\|X_{s-}^{(n)}\right\|_{H}^{p-4}\left\|\left(P_{n} B\left(s, X_{s}^{(n)}\right) \tilde{P}_{n}\right)^{*} X_{s-}^{(n)}\right\|_{H}^{2} \mathrm{~d} s \\
& +\frac{p}{2} \int_{0}^{t}\left\|X_{s-}^{(n)}\right\|_{H}^{p-2}\left(2_{V^{*}}\left\langle A\left(s, X_{s}^{(n)}\right), X_{s-}^{(n)}\right\rangle_{V}+\left\|P_{n} B\left(s, X_{s}^{(n)}\right) \tilde{P}_{n}\right\|_{2}^{2}\right) \mathrm{d} s \\
& +\int_{0}^{t} p\left\|X_{s-}^{(n)}\right\|_{H}^{p-2}\left\langle X_{s-}^{(n)}, P_{n} B\left(s, X_{s}^{(n)}\right) \mathrm{d} W_{s}^{(n)}\right\rangle_{H} \\
& +\int_{0}^{t} \int_{D^{c}} p\left\|X_{s-}^{(n)}\right\|_{H}^{p-2}\left\langle X_{s-}^{(n)}, P_{n} f\left(s, X_{s-}^{(n)}, z\right)\right\rangle_{H} \tilde{N}(\mathrm{~d} s, \mathrm{~d} z) \\
& +\int_{0}^{t} \int_{D^{c}}\left[\left\|X_{s-}^{(n)}+P_{n} f\left(s, X_{s-}^{(n)}, z\right)\right\|_{H}^{p}-\left\|X_{s-}^{(n)}\right\|_{H}^{p}\right. \\
& \left.\quad-p\left\|X_{s-}^{(n)}\right\|_{H}^{p-2}\left\langle X_{s-}^{(n)}, P_{n} f\left(s, X_{s-}^{(n)}, z\right)\right\rangle_{H}\right] N(\mathrm{~d} s, \mathrm{~d} z), \quad t \in[0, T] .
\end{aligned}
$$

Then assumption $(H 3)$ and (1.2) imply that

$$
\begin{aligned}
& \left\|X_{t}^{(n)}\right\|_{H}^{p}+\frac{p \theta}{2} \int_{0}^{t}\left\|X_{s}^{(n)}\right\|_{H}^{p-2}\left\|X_{s}^{(n)}\right\|_{V}^{\alpha} \mathrm{d} s \\
\leq & \|x\|_{H}^{p}+p(p-2) \int_{0}^{t}\left(C\left\|X_{s}^{(n)}\right\|_{H}^{p}+F_{s} \cdot\left\|X_{s}^{(n)}\right\|_{H}^{p-2}+\gamma\left\|X_{s}^{(n)}\right\|_{H}^{p-2}\left\|X_{s}^{(n)}\right\|_{V}^{\alpha}\right) \mathrm{d} s \\
& +\frac{p}{2} \int_{0}^{t}\left(K\left\|X_{s}^{(n)}\right\|_{H}^{p}+F_{s} \cdot\left\|X_{s}^{(n)}\right\|_{H}^{p-2}\right) \mathrm{d} s+Y(t)+Z(t)+I(t),
\end{aligned}
$$


where $Y, Z, I$ are processes defined by, for $t \geq 0$,

$$
\begin{aligned}
Y(t)= & \int_{0}^{t} p\left\|X_{s-}^{(n)}\right\|_{H}^{p-2}\left\langle X_{s-}^{(n)}, P_{n} B\left(s, X_{s}^{(n)}\right) \mathrm{d} W_{s}^{(n)}\right\rangle_{H} ; \\
Z(t)= & \int_{0}^{t} \int_{D^{c}} p\left\|X_{s-}^{(n)}\right\|_{H}^{p-2}\left\langle X_{s-}^{(n)}, P_{n} f\left(s, X_{s-}^{(n)}, z\right)\right\rangle_{H} \tilde{N}(\mathrm{~d} s, \mathrm{~d} z) ; \\
I(t)= & \int_{0}^{t} \int_{D^{c}} \mid\left\|X_{s-}^{(n)}+P_{n} f\left(s, X_{s-}^{(n)}, z\right)\right\|_{H}^{p}-\left\|X_{s-}^{(n)}\right\|_{H}^{p} \\
& -p\left\|X_{s-}^{(n)}\right\|_{H}^{p-2}\left\langle X_{s-}^{(n)}, P_{n} f\left(s, X_{s-}^{(n)}, z\right)\right\rangle_{H} \mid N(\mathrm{~d} s, \mathrm{~d} z) .
\end{aligned}
$$

Note that $\left\|X_{t}^{(n)}\right\|_{H} \leq R$, for $t<\tau_{R}^{(n)}$ and $X_{t}^{(n)}$ takes values in $H_{n}$. Hence there exists a constant $C$ such that

$$
\left\|X_{t}^{(n)}\right\|_{V} \leq C R, \quad t<\tau_{R}^{(n)}
$$

Thus by (1.2) we have

$$
\begin{gathered}
\mathbb{E} \int_{0}^{t \wedge \tau_{R}^{(n)}}\left\|X_{s-}^{(n)}\right\|_{H}^{2(p-1)}\left\|B\left(s, X_{s}^{(n)}\right)\right\|_{2}^{2} \mathrm{~d} s<\infty \\
\mathbb{E} \int_{0}^{t \wedge \tau_{R}^{(n)}} \int_{D^{c}}\left\|X_{s-}^{(n)}\right\|_{H}^{2(p-1)}\left\|f\left(s, X_{s-}^{(n)}, z\right)\right\|_{H}^{2} \nu(\mathrm{d} z) \mathrm{d} s<\infty .
\end{gathered}
$$

Therefore, the stopped processes $Y_{t \wedge \tau_{R}^{(n)}}$ and $Z_{t \wedge \tau_{R}^{(n)}}$ are martingales. Denote, for notational simplicity, the stopped process $X_{t \wedge \tau_{R}^{(n)}}^{(n)}$ etc again by $X_{t}^{(n)}$ etc. Then by Young's inequality and martingale property we have

$$
\begin{aligned}
& \mathbb{E}\left\|X_{t}^{(n)}\right\|_{H}^{p}+\left(\frac{p \theta}{2}-\gamma p(p-2)\right) \mathbb{E} \int_{0}^{t}\left\|X_{s}^{(n)}\right\|_{H}^{p-2}\left\|X_{s}^{(n)}\right\|_{V}^{\alpha} \mathrm{d} s \\
\leq & \mathbb{E}\|x\|_{H}^{p}+C \mathbb{E} \int_{0}^{t}\left(\left\|X_{s}^{(n)}\right\|_{H}^{p}+F_{s}^{p / 2}\right) \mathrm{d} s+\mathbb{E} I(t),
\end{aligned}
$$

where $C$ is some constant.

Define $g(t):=\|x+t h\|_{H}^{p}$, then by applying the Taylor formula to $g$ (cf. e.g. [4]) we can get that for some constant $C_{p}(p \geq 2)$

$$
\begin{aligned}
\left|\|x+h\|_{H}^{p}-\|x\|_{H}^{p}-p\|x\|_{H}^{p-2}\langle x, h\rangle_{H}\right| & =p\left|\int_{0}^{1}\left[\|x+t h\|_{H}^{p-2}\langle x+t h, h\rangle_{H}-\|x\|_{H}^{p-2}\langle x, h\rangle_{H}\right] \mathrm{d} t\right| \\
& \leq C_{p} \int_{0}^{1}\left(\|x\|_{H}+\|h\|_{H}\right)^{p-2}|h|_{H}^{2} t \mathrm{~d} t \\
& \leq C_{p}\left(\|x\|_{H}^{p-2}\|h\|_{H}^{2}+\|h\|_{H}^{p}\right), x, h \in H_{n} .
\end{aligned}
$$

In particular, if $p=2$, the above inequality can be replaced by the equality with $C_{p}=1$, i.e.

$$
\left|\|x+h\|_{H}^{2}-\|x\|_{H}^{2}-2\langle x, h\rangle_{H}\right|=\|h\|_{H}^{2}, \text { for all } x, h \in H_{n} .
$$


Thus it follows from (3.9) and (1.3) that

$$
\begin{aligned}
\mathbb{E} I(t) \leq \mathbb{E} \int_{0}^{t} \int_{D^{c}} \mid\left\|X_{s-}^{(n)}+P_{n} f\left(s, X_{s-}^{(n)}, z\right)\right\|_{H}^{p}-\left\|X_{s-}^{(n)}\right\|_{H}^{p} \\
\quad-p\left\|X_{s-}^{(n)}\right\|_{H}^{p-2}\left\langle X_{s-}^{(n)}, P_{n} f\left(s, X_{s-}^{(n)}, z\right)\right\rangle_{H} \mid N(\mathrm{~d} s, \mathrm{~d} z) \\
=\mathbb{E} \int_{0}^{t} \int_{D^{c}} \mid\left\|X_{s}^{(n)}+P_{n} f\left(s, X_{s}^{(n)}, z\right)\right\|_{H}^{p}-\left\|X_{s}^{(n)}\right\|_{H}^{p} \\
\quad-p\left\|X_{s}^{(n)}\right\|^{p-2}\left\langle X_{s}^{(n)}, P_{n} f\left(s, X_{s}^{(n)}, z\right)\right\rangle_{H} \mid \nu(\mathrm{d} z) \mathrm{d} s \\
\leq C \mathbb{E} \int_{0}^{t}\left\|X_{s}^{(n)}\right\|_{H}^{p-2}\left\|f\left(s, X_{s}^{(n)}, z\right)\right\|_{H}^{2} \mathrm{~d} s+C \mathbb{E} \int_{0}^{t}\left\|f\left(s, X_{s}^{(n)}, z\right)\right\|_{H}^{p} \mathrm{~d} s \\
\leq C \mathbb{E} \int_{0}^{t}\left(F_{t}^{p / 2}+\left\|X_{s}^{(n)}\right\|_{H}^{p}\right) \mathrm{d} s,
\end{aligned}
$$

where $C$ is a generic constant.

Combining the above estimates we get

$$
\begin{aligned}
& \mathbb{E}\left\|X_{t}^{(n)}\right\|_{H}^{p}+\left(\frac{p \theta}{2}-\gamma p(p-2)\right) \mathbb{E} \int_{0}^{t}\left\|X_{s}^{(n)}\right\|_{H}^{p-2}\left\|X_{s}^{(n)}\right\|_{V}^{\alpha} \mathrm{d} s \\
\leq & \mathbb{E}\|x\|_{H}^{p}+C \mathbb{E} \int_{0}^{t}\left(\left\|X_{s}^{(n)}\right\|_{H}^{p}+F_{s}^{p / 2}\right) \mathrm{d} s
\end{aligned}
$$

where $C$ is some constant.

By the Gronwall Lemma we have some constant $C>0$ such that for any $R \geq 0$ and any $n \geq 1$

$$
\mathbb{E}\left\|X_{t \wedge \tau_{R}^{(n)}}^{(n)}\right\|_{H}^{p}+\mathbb{E} \int_{0}^{T \wedge \tau_{R}^{(n)}}\left\|X_{s}^{(n)}\right\|_{H}^{p-2}\left\|X_{s}^{(n)}\right\|_{V}^{\alpha} \mathrm{d} s \leq C\left(\mathbb{E}\|x\|_{H}^{p}+\mathbb{E} \int_{0}^{T} F_{s}^{p / 2} \mathrm{~d} s\right), t \geq 0 .
$$

Here the constant $C$ is independent of $n$ and the stopping times $\tau_{R}^{(n)}$. Therefore, by applying the Fatou Lemma we get the desired inequality (3.6).

If we assume the assumptions of Theorem 1.2, but with the condition (1.3) replaced by a weaker assumption

$$
\int_{D^{c}}\|f(t, v, z)\|_{H}^{\beta+2} \nu(\mathrm{d} z) \leq F_{t}^{(\beta+2) / 2}+C\|v\|_{H}^{\beta+2}+\gamma\|v\|_{H}^{\beta}\|v\|_{V}^{\alpha}
$$

we arrive at the following Lemma.

Lemma 3.3. There exists a constant $\gamma_{0}$ such that if (3.11) is satisfied with $\gamma<\gamma_{0}$, then we have

$$
\sup _{n \in \mathbb{N}}\left(\mathbb{E} \sup _{t \in[0, T]}\left\|X_{t}^{(n)}\right\|_{H}^{\beta+2}+\mathbb{E} \int_{0}^{T}\left\|X_{t}^{(n)}\right\|_{H}^{\beta}\left\|X_{t}^{(n)}\right\|_{V}^{\alpha} \mathrm{d} t\right) \leq C\left(\mathbb{E}\|x\|_{H}^{\beta+2}+\mathbb{E} \int_{0}^{T} F_{t}^{(\beta+2) / 2} \mathrm{~d} t\right) .
$$


Proof. Let $p=\beta+2$ as before. By (3.8), (H3) and (1.2), we find

$$
\begin{aligned}
& \sup _{s \in\left[0, t \wedge \tau_{R}^{(n)}\right]}\left\|X_{s}^{(n)}\right\|_{H}^{p}+\frac{p \theta}{2} \int_{0}^{t \wedge \tau_{R}^{(n)}}\left\|X_{s}^{(n)}\right\|_{H}^{p-2}\left\|X_{s}^{(n)}\right\|_{V}^{\alpha} \mathrm{d} s \\
& \leq\|x\|_{H}^{p}+p(p-2) \int_{0}^{t \wedge \tau_{R}^{(n)}}\left(C\left\|X_{s}^{(n)}\right\|_{H}^{p}+F_{s} \cdot\left\|X_{s}^{(n)}\right\|_{H}^{p-2}+\gamma\left\|X_{s}^{(n)}\right\|_{H}^{p-2}\left\|X_{s}^{(n)}\right\|_{V}^{\alpha}\right) \mathrm{d} s \\
& \quad+\frac{p}{2} \int_{0}^{t \wedge \tau_{R}^{(n)}}\left(K\left\|X_{s}^{(n)}\right\|_{H}^{p}+F_{s} \cdot\left\|X_{s}^{(n)}\right\|_{H}^{p-2}\right) \mathrm{d} s+I_{1}(t)+I_{2}(t)+I_{3}(t) \\
& \leq\|x\|_{H}^{p}+\gamma p(p-2) \int_{0}^{t \wedge \tau_{R}^{(n)}}\left\|X_{s}^{(n)}\right\|_{H}^{p-2}\left\|X_{s}^{(n)}\right\|_{V}^{\alpha} \mathrm{d} s \\
& \quad+C \int_{0}^{t \wedge \tau_{R}^{(n)}}\left(\left\|X_{s}^{(n)}\right\|_{H}^{p}+F_{s}^{p / 2}\right) \mathrm{d} s+I_{1}(t)+I_{2}(t)+I_{3}(t),
\end{aligned}
$$

where $C$ is a generic constant, $\tau_{R}^{(n)}$ are the stopping times defined in (3.7) and $I_{1}, I_{2}, I_{3}$ are processes defined by, for $t \geq 0$,

$$
\begin{aligned}
& I_{1}(t):=p \sup _{r \in\left[0, t \wedge \tau_{R}^{(n)}\right]}\left|\int_{0}^{r}\left\|X_{s}^{(n)}\right\|_{H}^{p-2}\left\langle X_{s}^{(n)}, P_{n} B\left(s, X_{s}^{(n)}\right) \mathrm{d} W_{s}^{(n)}\right\rangle_{H}\right| ; \\
& I_{2}(t):=p \sup _{r \in\left[0, t \wedge \tau_{R}^{(n)}\right]}\left|\int_{0}^{r} \int_{D^{c}}\left\|X_{s}^{(n)}\right\|_{H}^{p-2}\left\langle X_{s-}^{(n)}, P_{n} f\left(s, X_{s-}^{(n)}, z\right)\right\rangle_{H} \tilde{N}(\mathrm{~d} s, \mathrm{~d} z)\right| ; \\
& I_{3}(t):=\sup _{r \in\left[0, t \wedge \tau_{R}^{(n)}\right]} \mid \int_{0}^{r} \int_{D^{c}}\left[\left\|X_{s-}^{(n)}+P_{n} f\left(s, X_{s-}^{(n)}, z\right)\right\|_{H}^{p}-\left\|X_{s-}^{(n)}\right\|_{H}^{p}\right. \\
& \left.\quad-p\left\|X_{s-}^{(n)}\right\|_{H}^{p-2}\left\langle X_{s-}^{(n)}, P_{n} f\left(s, X_{s-}^{(n)}, z\right)\right\rangle\right] N(\mathrm{~d} z, \mathrm{~d} s) \mid .
\end{aligned}
$$

On the basis of the Burkholder-Davis-Gundy inequality (cf.[30]), assumption (1.2), the Cauchy-Schwartz and the Young inequalities, we have for any $\varepsilon>0$,

$$
\begin{aligned}
& \mathbb{E} I_{1}(t) \\
= & p \mathbb{E} \sup _{r \in\left[0, t \wedge \tau_{R}^{(n)}\right]}\left|\int_{0}^{r}\left\|X_{s}^{(n)}\right\|_{H}^{p-2}\left\langle X_{s}^{(n)}, P_{n} B\left(s, X_{s}^{(n)}\right) \mathrm{d} W_{s}^{(n)}\right\rangle_{H}\right| \\
\leq & 3 p \mathbb{E}\left[\int_{0}^{t \wedge \tau_{R}^{(n)}}\left\|X_{s}^{(n)}\right\|_{H}^{2 p-2}\left\|B\left(s, X_{s}^{(n)}\right)\right\|_{2}^{2} \mathrm{~d} s\right]^{1 / 2} \\
\leq & 3 p \mathbb{E}\left[\sup _{s \in\left[0, t \wedge \tau_{R}^{(n)}\right]}\left\|X_{s}^{(n)}\right\|_{H}^{p} \cdot\left(\int_{0}^{t \wedge \tau_{R}^{(n)}}\left\|X_{s}^{(n)}\right\|_{H}^{p-2}\left(F_{s}+C\left\|X_{s}^{(n)}\right\|_{H}^{2}+\gamma\left\|X_{s}^{(n)}\right\|_{V}^{\alpha}\right) \mathrm{d} s\right)\right]^{1 / 2}
\end{aligned}
$$




$$
\begin{aligned}
& \leq 3 p\left[\varepsilon \mathbb{E} \sup _{s \in[0, t]}\left\|X_{s}^{(n)}\right\|_{H}^{p}\right]^{1 / 2}\left[\frac{1}{\varepsilon} \mathbb{E}\left(\int_{0}^{t \wedge \tau_{R}^{(n)}}\left\|X_{s}^{(n)}\right\|_{H}^{p-2}\left(F_{s}+C\left\|X_{s}^{(n)}\right\|_{H}^{2}+\gamma\left\|X_{s}^{(n)}\right\|_{V}^{\alpha}\right) \mathrm{d} s\right)\right]^{1 / 2} \\
& \leq \varepsilon \mathbb{E} \sup _{s \in\left[0, t \wedge \tau_{R}^{(n)}\right]}\left\|X_{s}^{(n)}\right\|_{H}^{p}+C_{\varepsilon, p} \mathbb{E}\left(\int_{0}^{t \wedge \wedge \tau_{R}^{(n)}}\left\|X_{s}^{(n)}\right\|_{H}^{p-2}\left(F_{s}+C\left\|X_{s}^{(n)}\right\|_{H}^{2}+\gamma\left\|X_{s}^{(n)}\right\|_{V}^{\alpha}\right) \mathrm{d} s\right) \\
& \leq \varepsilon \mathbb{E} \sup _{s \in\left[0, t \wedge \tau_{R}^{(n)}\right]}\left\|X_{s}^{(n)}\right\|_{H}^{p}+\gamma C_{\varepsilon, p} \mathbb{E} \int_{0}^{t \wedge \tau_{R}^{(n)}}\left\|X_{s}^{(n)}\right\|_{H}^{p-2}\left\|X_{s}^{(n)}\right\|_{V}^{\alpha} d s \\
& \quad+C_{\varepsilon, p} \mathbb{E} \int_{0}^{t \wedge \tau_{R}^{(n)}}\left(\left\|X_{s}^{(n)}\right\|_{H}^{p}+F_{s}^{p / 2}\right) \mathrm{d} s .
\end{aligned}
$$

Similarly, using (1.2), the Burkholder-Davis inequality and the Young inequality we have

$$
\begin{aligned}
& \mathbb{E} I_{2}(t) \\
= & p \mathbb{E} \sup _{r \in\left[0, t \wedge \tau_{R}^{(n)}\right]}\left|\int_{0}^{r} \int_{D^{c}}\left\|X_{s}^{(n)}\right\|_{H}^{p-2}\left\langle X_{s-}^{(n)}, P_{n} f\left(s, X_{s-}^{(n)}, z\right)\right\rangle_{H} \tilde{N}(\mathrm{~d} s, \mathrm{~d} z)\right| \\
\leq & C \mathbb{E}\left[\int_{0}^{t \wedge \tau_{R}^{(n)}} \int_{D^{c}}\left\|X_{s}^{(n)}\right\|_{H}^{2 p-2}\left\|P_{n} f\left(s, X_{s}^{(n)}, z\right)\right\|_{H}^{2} \nu(\mathrm{d} z) \mathrm{d} s\right]^{\frac{1}{2}} \\
\leq & C \mathbb{E}\left[\sup _{s \in\left[0, t \wedge \tau_{R}^{(n)}\right]}\left\|X_{s}^{(n)}\right\|_{H}^{p}\left(\int_{0}^{t \wedge \tau_{R}^{(n)}}\left\|X_{s}^{(n)}\right\|_{H}^{p-2}\left(F_{s}+C\left\|X_{s}^{(n)}\right\|_{H}^{2}+\gamma\left\|X_{s}^{(n)}\right\|_{V}^{\alpha}\right) \mathrm{d} s\right)\right]^{\frac{1}{2}} \\
\leq & \varepsilon \mathbb{E} \sup _{s \in\left[0, t \wedge \tau_{R}^{(n)}\right]}\left\|X_{s}^{(n)}\right\|_{H}^{p}+C_{\varepsilon, p} \mathbb{E}\left(\int_{0}^{t \wedge \tau_{R}^{(n)}}\left\|X_{s}^{(n)}\right\|^{p-2}\left(F_{s}+\left\|X_{s}^{(n))}\right\|_{H}^{2}+\gamma\left\|X_{s}^{(n))}\right\|_{V}^{\alpha}\right) \mathrm{d} s\right) \\
\leq & \varepsilon \mathbb{E} \sup _{s \in\left[0, t \wedge \tau_{R}^{(n)}\right]}\left\|X_{s}^{(n)}\right\|_{H}^{p}+\gamma C_{\varepsilon, p} \mathbb{E} \int_{0}^{t \wedge \tau_{R}^{(n)}}\left\|X_{s}^{(n)}\right\|_{H}^{p-2}\left\|X_{s}^{(n)}\right\|_{V}^{\alpha} d s \\
+ & C_{\varepsilon, p} \mathbb{E} \int_{0}^{t \wedge \tau_{R}^{(n)}}\left(\left\|X_{s}^{(n)}\right\|_{H}^{p}+F_{s}^{p / 2}\right) \mathrm{d} s,
\end{aligned}
$$

where $C_{\varepsilon, p}$ is not necessarily the same number from line to line.

For the term $I_{3}(t)$, by (3.11), (1.2) and (3.9), we have

$$
\begin{aligned}
\mathbb{E} I_{3}(t) \leq \mathbb{E} \int_{0}^{t \wedge \tau_{R}^{(n)}} \int_{D^{c}} \mid\left\|X_{s-}^{(n)}+P_{n} f\left(s, X_{s-}^{(n)}, z\right)\right\|_{H}^{p}-\left\|X_{s-}^{(n)}\right\|_{H}^{p} \\
\quad-p\left\|X_{s-}^{(n)}\right\|_{H}^{p-2}\left\langle X_{s-}^{(n)}, P_{n} f\left(s, X_{s-}^{(n)}, z\right)\right\rangle_{H} \mid N(\mathrm{~d} s, \mathrm{~d} z) \\
=\mathbb{E} \int_{0}^{t \wedge \tau_{R}^{(n)}} \int_{D^{c}} \mid\left\|X_{s}^{(n)}+P_{n} f\left(s, X_{s}^{(n)}, z\right)\right\|_{H}^{p}-\left\|X_{s}^{(n)}\right\|_{H}^{p}
\end{aligned}
$$




$$
\begin{gathered}
\quad p\left\|X_{s}^{(n)}\right\|^{p-2}\left\langle X_{s}^{(n)}, P_{n} f\left(s, X_{s}^{(n)}, z\right)\right\rangle_{H} \mid \nu(\mathrm{d} z) \mathrm{d} s \\
\leq C_{p} \mathbb{E} \int_{0}^{t \wedge \tau_{R}^{(n)}} \int_{D^{c}}\left(\left\|X_{s}^{(n)}\right\|_{H}^{p-2}\left\|f\left(s, X_{s}^{(n)}, z\right)\right\|_{H}^{2}+\left\|f\left(s, X_{s}^{(n)}, z\right)\right\|_{H}^{p}\right) \nu(\mathrm{d} z) \mathrm{d} s \\
\leq \gamma C_{p} \mathbb{E} \int_{0}^{t \wedge \tau_{R}^{(n)}}\left\|X_{s}^{(n)}\right\|_{H}^{p-2}\left\|X_{s}^{(n)}\right\|_{V}^{\alpha} \mathrm{d} s+C_{p} \mathbb{E} \int_{0}^{t \wedge \tau_{R}^{(n)}}\left(F_{t}^{p / 2}+\left\|X_{s}^{(n)}\right\|_{H}^{p}\right) \mathrm{d} s .
\end{gathered}
$$

Combining the estimates (3.13) $-(3.15)$ we get

$$
\begin{aligned}
& \mathbb{E}\left(I_{1}(t)+I_{2}(t)+I_{3}(t)\right) \\
& \leq 2 \varepsilon \mathbb{E} \sup _{s \in\left[0, t \wedge \tau_{R}^{(n)}\right]}\left\|X_{s}^{(n)}\right\|_{H}^{p}+\gamma C_{\varepsilon, p} \mathbb{E} \int_{0}^{t \wedge \tau_{R}^{(n)}}\left\|X_{s}^{(n)}\right\|_{H}^{p-2}\left\|X_{s}^{(n)}\right\|_{V}^{\alpha} d s \\
& \quad+C_{\varepsilon, p} \mathbb{E} \int_{0}^{t \wedge \tau_{R}^{(n)}}\left\|X_{s}^{(n)}\right\|_{H}^{p} \mathrm{~d} s+C_{\varepsilon, p} \mathbb{E} \int_{0}^{T} F_{s}^{p / 2} \mathrm{~d} s .
\end{aligned}
$$

Let $\varepsilon=\frac{1}{3}$, then we have

$$
\begin{aligned}
& \frac{1}{3} \mathbb{E} \sup _{s \in\left[0, t \wedge \tau_{R}^{(n)}\right]}\left\|X_{s}^{(n)}\right\|_{H}^{p}+\left(\frac{p \theta}{2}-3 \gamma C_{0}\right) \mathbb{E} \int_{0}^{t \wedge \tau_{R}^{(n)}}\left\|X_{s}^{(n)}\right\|_{H}^{p-2}\left\|X_{s}^{(n)}\right\|_{V}^{\alpha} \mathrm{d} s \\
\leq & \mathbb{E}\|x\|_{H}^{p}+C_{0} \mathbb{E} \int_{0}^{t \wedge \tau_{R}^{(n)}}\left\|X_{s}^{(n)}\right\|_{H}^{p} \mathrm{~d} s+C_{0} \mathbb{E} \int_{0}^{T} F_{s}^{p / 2} \mathrm{~d} s,
\end{aligned}
$$

where $C_{0}$ is some constant.

Observe that $\left\|X_{s}^{(n)}\right\|_{H} \leq R$, for $s<\tau_{R}^{(n)}$. Then we see that the right-hand side of the above inequality is finite. Therefore, if $\gamma$ is small enough (e.g. $\gamma<\gamma_{0}:=\frac{p \theta}{6 C_{0}}$ ), we may apply the Gronwall Lemma to infer that there exists $C>0$ such that for any $n \geq 1$

$$
\mathbb{E} \sup _{t \in\left[0, T \wedge \tau_{R}^{(n)}\right]}\left\|X_{t}^{(n)}\right\|_{H}^{p}+\mathbb{E} \int_{0}^{T \wedge \tau_{R}^{(n)}}\left\|X_{s}^{(n)}\right\|_{H}^{p-2}\left\|X_{s}^{(n)}\right\|_{V}^{\alpha} \mathrm{d} s \leq C\left(\mathbb{E}\|x\|_{H}^{p}+\mathbb{E} \int_{0}^{T} F_{s}^{p / 2} \mathrm{~d} s\right) .
$$

Recall that $\tau_{R}^{(n)} \uparrow T, \mathbb{P}$-a.s. and $\mathbb{P}\left\{\tau_{R}^{(n)}<T\right\}=0$ as $R \rightarrow \infty$. Therefore, by the Fatou Lemma we obtain that

$$
\begin{aligned}
& \mathbb{E} \sup _{t \in[0, T]}\left\|X_{t}^{(n)}\right\|_{H}^{p}+\mathbb{E} \int_{0}^{T}\left\|X_{s}^{(n)}\right\|_{H}^{p-2}\left\|X_{s}^{(n)}\right\|_{V}^{\alpha} \mathrm{d} s \\
& \leq \liminf _{R \rightarrow \infty}\left(\mathbb{E} \sup _{t \in\left[0, T \wedge \tau_{R}^{(n)}\right]}\left\|X_{t}^{(n)}\right\|_{H}^{p}+\mathbb{E} \int_{0}^{T \wedge \tau_{R}^{(n)}}\left\|X_{s}^{(n)}\right\|_{H}^{p-2}\left\|X_{s}^{(n)}\right\|_{V}^{\alpha} \mathrm{d} s\right) \\
& \leq C\left(\mathbb{E}\left\|X_{0}\right\|_{H}^{p}+\mathbb{E} \int_{0}^{T} F_{s}^{p / 2} \mathrm{~d} s\right), \text { for all } n \geq 1 \text {. }
\end{aligned}
$$

This completes the proof of Lemma 3.3 . 
For the simplicity of notations, we introduce the following three auxiliary spaces:

$$
\begin{aligned}
& K=L^{\alpha}([0, T] \times \Omega, \mathrm{d} t \times \mathbb{P} ; V) \\
& J=L^{2}\left([0, T] \times \Omega, \mathrm{d} t \times \mathbb{P} ; \mathcal{T}_{2}(U ; H)\right) \\
& \mathcal{M}=\mathcal{M}_{T}^{2}(\mathcal{P} \otimes \mathcal{Z}, \mathrm{d} t \times \mathbb{P} \times \nu ; H)
\end{aligned}
$$

Note that $K^{*}=L^{\frac{\alpha}{\alpha-1}}\left([0, T] \times \Omega, \mathrm{d} t \times \mathbb{P} ; V^{*}\right)$.

Lemma 3.4. Under the assumptions of Theorem 1.2, there exists a subsequence $\left(n_{k}\right)$ and an element $\bar{X} \in K \cap L^{\infty}\left([0, T] ; L^{p}(\Omega ; H)\right)$ such that

(i) $X^{\left(n_{k}\right)} \rightarrow \bar{X}$ weakly in $K$ and weakly star in $L^{\infty}\left([0, T] ; L^{p}(\Omega ; H)\right)$;

(ii) $Y^{\left(n_{k}\right)}:=P_{n_{k}} A\left(\cdot, X^{\left(n_{k}\right)}\right) \rightarrow Y$ weakly in $K^{*}$;

(iii) $Z^{\left(n_{k}\right)}:=P_{n_{k}} B\left(\cdot, X^{\left(n_{k}\right)}\right) \rightarrow Z$ weakly in $J$ and

$$
\int_{0}^{\cdot} P_{n_{k}} B\left(s, X_{s}^{\left(n_{k}\right)}\right) \mathrm{d} W_{s}^{\left(n_{k}\right)} \rightarrow \int_{0}^{\cdot} Z_{s} \mathrm{~d} W_{s}
$$

weakly in $L^{\infty}\left([0, T], \mathrm{d} t ; L^{2}(\Omega, \mathbb{P} ; H)\right)$;

(iv) $F^{\left(n_{k}\right)}:=P_{n_{k}} f\left(\cdot, X^{\left(n_{k}\right)}, \cdot\right) 1_{D^{c}} \rightarrow F 1_{D^{c}}$ weakly in $\mathcal{M}$.

Proof. Applying Lemma 3.2 with $p=2$ (instead of taking $p=\beta+2$ ) we have

$$
\sup _{n} \mathbb{E} \int_{0}^{T}\left\|X_{t}^{(n)}\right\|_{V}^{\alpha} d t<\infty .
$$

Since the space $\mathrm{K}$ is reflexive, we can find a weakly convergent subsequence $\left\{X^{\left(n_{k}\right)}\right\}$ and $\bar{X} \in K$ such that $X^{\left(n_{k}\right)}$ converges to $\bar{X}$ weakly in $K$.

Similarly, since $L^{\infty}\left([0, T] ; L^{p}(\Omega ; H)\right)=\left(L^{1}\left([0, T] ; L^{\frac{p}{p-1}}(\Omega ; H)\right)\right)^{*}$, by the Banach-Alaoglu Theorem, (3.6) allows us to get another weakly* convergent subsequence (for simplicity we still denote it by the same notation $\left.\left\{X^{\left(n_{k}\right)}\right\}\right)$ and $\bar{X} \in K \cap L^{p}\left(\Omega ; L^{\infty}([0, T] ; H)\right)$ such that assertion $(i)$ holds. Meanwhile, by $(H 4)$ and (3.6) we have

$$
\begin{aligned}
& \sup _{n} \mathbb{E} \int_{0}^{T}\left\|A\left(t, X_{t}^{(n)}\right)\right\|_{V^{*}}^{\frac{\alpha}{\alpha-1}} \mathrm{~d} t \\
\leq & \sup _{n} \mathbb{E} \int_{0}^{T}\left(F_{t}+C\left\|X_{t}^{(n)}\right\|_{V}^{\alpha}\right)\left(1+\left\|X_{t}^{(n)}\right\|_{H}^{\beta}\right) \mathrm{d} t \\
\leq & C \sup _{n} \mathbb{E} \int_{0}^{T}\left(F_{t}+\left\|X_{t}^{(n)}\right\|_{V}^{\alpha}+F_{t}^{\frac{\beta+2}{2}}+\left\|X_{t}^{(n)}\right\|_{H}^{\beta+2}+\left\|X_{t}^{(n)}\right\|_{H}^{\beta}\left\|X_{t}^{(n)}\right\|_{V}^{\alpha}\right) \mathrm{d} t<\infty .
\end{aligned}
$$

Therefore, claim (ii) also holds.

Also, note that by (1.2)

$$
\begin{aligned}
& \sup _{n} \mathbb{E} \int_{0}^{T}\left\|P_{n} B\left(t, X_{t}^{(n)}\right)\right\|_{2}^{2} \mathrm{~d} t \\
\leq & \sup _{n} \mathbb{E} \int_{0}^{T}\left(F_{t}+C\left\|X_{t}^{(n)}\right\|_{H}^{2}+\gamma\left\|X_{t}^{(n)}\right\|_{V}^{\alpha}\right) \mathrm{d} t<\infty .
\end{aligned}
$$


Hence by taking a subsequence we have that $P_{n_{k}} B\left(t, X_{t}^{\left(n_{k}\right)}\right)$ converges to $Z$ weakly in $J$.

Recall that $\tilde{P}_{n}$ is the orthogonal projection in $U$ onto $\operatorname{span}\left\{g_{1}, \cdots, g_{n}\right\}$. Hence, without loss of generality we can assume that $P_{n_{k}} B\left(t, X_{t}^{\left(n_{k}\right)}\right) \tilde{P}_{n}$ also converges to $Z$ weakly in $J$. Since

$$
\int_{0}^{\cdot} P_{n} B\left(s, X_{t}^{\left(n_{k}\right)}\right) \mathrm{d} W_{s}^{n_{k}}=\int_{0}^{\cdot} P_{n_{k}} B\left(s, X_{s}^{\left(n_{k}\right)}\right) \tilde{P}_{n_{k}} \mathrm{~d} W_{s}
$$

weak convergence is preserved under the linear continuous maps and the map

$$
I: \phi \in J \mapsto I(\phi):=\int \phi \mathrm{d} W \in L^{2}([0, T] \times \Omega ; H)
$$

is continuous, we infer that

$$
\int_{0}^{\cdot} P_{n} B\left(s, X_{s}^{\left(n_{k}\right)}\right) \tilde{P}_{n} \mathrm{~d} W_{s} \rightarrow \int_{0}^{\cdot} Z_{s} \mathrm{~d} W_{s} \text { weakly. }
$$

Hence (iii) holds.

Similarly, by (1.2) we have

$$
\begin{aligned}
& \sup _{n} \mathbb{E} \int_{0}^{T} \int_{D^{c}}\left\|P_{n} f\left(s, X_{s-}^{(n)}, z\right)\right\|_{H}^{2} \nu(\mathrm{d} z) \mathrm{d} s \\
\leq & \sup _{n} \int_{0}^{T}\left(F_{t}+C\left\|X_{s}^{(n)}\right\|_{H}^{2}+\gamma\left\|X_{s}^{(n)}\right\|_{V}^{\alpha}\right) \mathrm{d} s<\infty,
\end{aligned}
$$

which yields claim $(i v)$.

Proof of Theorem 3.1. Existence of solutions: Let us define a $V^{*}$-valued process $X$ by

$$
X_{t}:=X_{0}+\int_{0}^{t} Y_{s} \mathrm{~d} s+\int_{0}^{t} Z_{s} \mathrm{~d} W_{s}+\int_{0}^{t} \int_{D^{c}} F(s, z) \tilde{N}(\mathrm{~d} s, \mathrm{~d} z), t \in[0, T] .
$$

By Lemma 3.4, it is easy to see that $X$ is a $V^{*}$-valued modification of the $V$-valued process $\bar{X}$, i.e. $X=\bar{X} \mathrm{~d} t \times \mathbb{P}$-a.e.. Moreover, we have

$$
\sup _{t \in[0, T]} \mathbb{E}\left\|X_{t}\right\|_{H}^{p}+\mathbb{E} \int_{0}^{T}\left\|X_{t}\right\|_{V}^{\alpha} \mathrm{d} t<\infty
$$

By [23], we infer that $X$ is an $H$-valued càdlàg $\mathbb{F}$-adapted process satisfying

$$
\begin{aligned}
\left\|X_{t}\right\|_{H}^{2}= & \left\|X_{0}\right\|_{H}^{2}+\int_{0}^{t}\left(2_{V^{*}}\left\langle Y_{s}, \bar{X}_{s}\right\rangle_{V}+\left\|Z_{s}\right\|_{2}^{2}\right) \mathrm{d} s+2 \int_{0}^{t}\left\langle\bar{X}_{s}, Z_{s} \mathrm{~d} W_{s}\right\rangle_{H} \\
& +2 \int_{0}^{t} \int_{D^{c}}\left\langle\bar{X}_{s}, F(s, z)\right\rangle_{H} \tilde{N}(\mathrm{~d} s, \mathrm{~d} z)+\int_{0}^{t} \int_{D^{c}}\|F(s, z)\|_{H}^{2} N(\mathrm{~d} s, \mathrm{~d} z) .
\end{aligned}
$$


Therefore, in order to prove that $X$ is a solution of 3.1 it remains to verify that

$$
\begin{array}{r}
A(\cdot, \bar{X})=Y, B(\cdot, \bar{X})=Z, \quad \mathrm{~d} t \times \mathbb{P}-\text { a.e.; } \\
\text { and } f\left(s, \bar{X}_{s-}, z\right)=F(s, z), \quad \mathrm{d} t \times \mathbb{P} \times \nu-\text { a.e.. }
\end{array}
$$

Define

$$
\mathcal{N}=\left\{\phi: \phi \text { is a } V \text {-valued } \mathbb{F} \text {-adapted process such that } \mathbb{E} \int_{0}^{T} \rho\left(\phi_{s}\right) d s<\infty\right\} .
$$

For $\phi \in K \cap \mathcal{N} \cap L^{\infty}\left([0, T] ; L^{p}(\Omega ; H)\right)$, by applying the Itô formula to the process $X^{\left(n_{k}\right)}$, see Schmalfuss [54, proof of Theorem 4.1] and Temam [56] (for the deterministic case), we have

$$
\begin{aligned}
e^{-\int_{0}^{t}\left(K+\rho\left(\phi_{s}\right)\right) \mathrm{d} s}\left\|X_{t}^{\left(n_{k}\right)}\right\|_{H}^{2} \\
=\left\|X_{0}^{\left(n_{k}\right)}\right\|_{H}^{2}+\int_{0}^{t} e^{-\int_{0}^{s}\left(K+\rho\left(\phi_{r}\right)\right) \mathrm{d} r}\left(2_{V^{*}}\left\langle A\left(s, X_{s}^{\left(n_{k}\right)}\right), X_{s-}^{\left(n_{k}\right)}\right\rangle_{V}\right. \\
\left.\left.\quad+\left\|P_{n_{k}} B\left(s, X_{s}^{\left(n_{k}\right)}\right) \tilde{P}_{n_{k}}\right\|_{2}^{2}-\left(K+\rho\left(\phi_{s}\right)\right)\left\|X_{s}^{\left(n_{k}\right)}\right\|_{H}^{2}\right) \mathrm{~d} s\right] \\
\quad+2 \int_{0}^{t} e^{-\int_{0}^{s}\left(K+\rho\left(\phi_{r}\right)\right) \mathrm{d} r}\left\langle X_{s-}^{\left(n_{k}\right)}, P_{n_{k}} B\left(s, X_{s}^{\left(n_{k}\right)}\right) \mathrm{d} W_{s}^{n_{k}}\right\rangle_{H} \\
\quad+2 \int_{0}^{t} \int_{D^{c}} e^{-\int_{0}^{s}\left(K+\rho\left(\phi_{r}\right)\right) \mathrm{d} r}\left\langle X_{s-}^{\left(n_{k}\right)}, P_{n_{k}} f\left(s, X_{s-}^{\left(n_{k}\right)}, z\right)\right\rangle_{H} \tilde{N}(\mathrm{~d} s, \mathrm{~d} z) \\
\quad+\int_{0}^{t} \int_{D^{c}} e^{-\int_{0}^{s}\left(K+\rho\left(\phi_{r}\right)\right) \mathrm{d} r}\left\|P_{n_{k}} f\left(s, X_{s-}^{\left(n_{k}\right)}, z\right)\right\|_{H}^{2} N(\mathrm{~d} s, \mathrm{~d} z) .
\end{aligned}
$$

Thus, by taking the expectation of both sides of the above equality and $(H 2)$ we get

$$
\begin{aligned}
& \mathbb{E}\left(e^{-\int_{0}^{t}\left(K+\rho\left(\phi_{s}\right)\right) \mathrm{d} s}\left\|X_{t}^{\left(n_{k}\right)}\right\|_{H}^{2}\right)-\mathbb{E}\left(\left\|X_{0}^{\left(n_{k}\right)}\right\|_{H}^{2}\right) \\
= & \mathbb{E}\left[\int _ { 0 } ^ { t } e ^ { - \int _ { 0 } ^ { s } ( K + \rho ( \phi _ { r } ) ) \mathrm { d } r } \left(2_{V^{*}}\left\langle A\left(s, X_{s}^{\left(n_{k}\right)}\right), X_{s-}^{\left(n_{k}\right)}\right\rangle_{V}\right.\right. \\
& \left.\left.+\left\|P_{n_{k}} B\left(s, X_{s}^{\left(n_{k}\right)}\right) \tilde{P}_{n_{k}}\right\|_{2}^{2}-\left(K+\rho\left(\phi_{s}\right)\right)\left\|X_{s}^{\left(n_{k}\right)}\right\|_{H}^{2}\right) \mathrm{~d} s\right] \\
& +\mathbb{E}\left[\int_{0}^{t} \int_{D^{c}} e^{-\int_{0}^{s}\left(K+\rho\left(\phi_{r}\right)\right) \mathrm{d} r}\left\|P_{n_{k}} f\left(s, X_{s-}^{\left(n_{k}\right)}, z\right)\right\|_{H}^{2} \nu(\mathrm{d} z) \mathrm{d} s\right] \\
\leq & \mathbb{E}\left[\int _ { 0 } ^ { t } e ^ { - \int _ { 0 } ^ { s } ( K + \rho ( \phi _ { r } ) ) \mathrm { d } r } \left(2_{V^{*}}\left\langle A\left(s, X_{s}^{\left(n_{k}\right)}\right)-A\left(s, \phi_{s}\right), X_{s}^{\left(n_{k}\right)}-\phi_{s}\right\rangle_{V}\right.\right. \\
& +\left\|B\left(s, X_{s}^{\left(n_{k}\right)}\right)-B\left(s, \phi_{s}\right)\right\|_{2}^{2}-\left(K+\rho\left(\phi_{s}\right)\right)\left\|X_{s}^{\left(n_{k}\right)}-\phi_{s}\right\|_{H}^{2} \\
& \left.\left.+\int_{D^{c}}\left\|f\left(s, X_{s}^{\left(n_{k}\right)}, z\right)-f\left(s, \phi_{s}, z\right)\right\|_{H}^{2} \nu(\mathrm{d} z)\right) \mathrm{d} s\right] \\
& +\mathbb{E}\left[\int _ { 0 } ^ { t } e ^ { - \int _ { 0 } ^ { s } ( K + \rho ( \phi _ { r } ) ) \mathrm { d } r } \left(2_{V^{*}}\left\langle A\left(s, X_{s}^{\left(n_{k}\right)}\right)-A\left(s, \phi_{s}\right), \phi_{s}\right\rangle_{V}+2_{V^{*}}\left\langle A\left(s, \phi_{s}\right), X_{s}^{\left(n_{k}\right)}\right\rangle_{V}\right.\right.
\end{aligned}
$$




$$
\begin{aligned}
& -\left\|B\left(s, \phi_{s}\right)\right\|_{2}^{2}+2\left\langle B\left(s, X_{s}^{\left(n_{k}\right)}\right), B\left(s, \phi_{s}\right)\right\rangle_{\mathcal{T}_{2}(U, H)}-2\left(K+\rho\left(\phi_{s}\right)\right)\left\langle X_{s}^{\left(n_{k}\right)}, \phi_{s}\right\rangle_{H} \\
& \left.\left.+\left(K+\rho\left(\phi_{s}\right)\right)\left\|\phi_{s}\right\|_{H}^{2}+\int_{D^{c}}\left(2\left\langle f\left(s, X_{s}^{\left(n_{k}\right)}, z\right), f\left(s, \phi_{s}, z\right)\right\rangle_{H}-\left\|f\left(s, \phi_{s}, z\right)\right\|_{H}^{2}\right) \nu(\mathrm{d} z)\right) \mathrm{d} s\right] \\
\leq & \mathbb{E}\left[\int _ { 0 } ^ { t } e ^ { - \int _ { 0 } ^ { s } ( K + \rho ( \phi _ { r } ) ) \mathrm { d } r } \left(2_{V^{*}}\left\langle A\left(s, X_{s}^{\left(n_{k}\right)}\right)-A\left(s, \phi_{s}\right), \phi_{s}\right\rangle_{V}+2_{V^{*}}\left\langle A\left(s, \phi_{s}\right), X_{s}^{\left(n_{k}\right)}\right\rangle_{V}\right.\right. \\
& -\left\|B\left(s, \phi_{s}\right)\right\|_{2}^{2}+2\left\langle B\left(s, X_{s}^{\left(n_{k}\right)}\right), B\left(s, \phi_{s}\right)\right\rangle_{\mathcal{T}_{2}(U, H)}-2\left(K+\rho\left(\phi_{s}\right)\right)\left\langle X_{s}^{\left(n_{k}\right)}, \phi_{s}\right\rangle_{H} \\
& \left.\left.+\left(K+\rho\left(\phi_{s}\right)\right)\left\|\phi_{s}\right\|_{H}^{2}+\int_{D^{c}}\left(2\left\langle f\left(s, X_{s}^{\left(n_{k}\right)}, z\right), f\left(s, \phi_{s}, z\right)\right\rangle_{H}-\left\|f\left(s, \phi_{s}, z\right)\right\|_{H}^{2}\right) \nu(\mathrm{d} z)\right) \mathrm{d} s\right] .
\end{aligned}
$$

Hence for any nonnegative function $\psi \in L^{\infty}([0, T] ; \mathrm{d} t)$ we have

$$
\begin{aligned}
& \mathbb{E}\left[\int_{0}^{T} \psi_{t}\left(e^{-\int_{0}^{t}\left(K+\rho\left(\phi_{s}\right)\right) \mathrm{d} s}\left\|X_{t}\right\|_{H}^{2}-\left\|X_{0}\right\|_{H}^{2}\right) \mathrm{d} t\right] \\
& \leq \liminf _{k \rightarrow \infty} \mathbb{E}\left[\int_{0}^{T} \psi_{t}\left(e^{-\int_{0}^{t}\left(K+\rho\left(\phi_{s}\right)\right) \mathrm{d} s}\left\|X_{t}^{\left(n_{k}\right)}\right\|_{H}^{2}-\left\|X_{0}^{\left(n_{k}\right)}\right\|_{H}^{2}\right) \mathrm{d} t\right] \\
& \leq \liminf _{k \rightarrow \infty} \mathbb{E}\left[\int _ { 0 } ^ { T } \psi _ { t } \left(\int _ { 0 } ^ { t } e ^ { - \int _ { 0 } ^ { s } ( K + \rho ( \phi _ { r } ) ) \mathrm { d } r } \left(2_{V^{*}}\left\langle A\left(s, X_{s}^{\left(n_{k}\right)}\right)-A\left(s, \phi_{s}\right), \phi_{s}\right\rangle_{V}\right.\right.\right. \\
& +2_{V^{*}}\left\langle A\left(s, \phi_{s}\right), X_{s}^{\left(n_{k}\right)}\right\rangle_{V}-\left\|B\left(s, \phi_{s}\right)\right\|_{2}^{2}+2\left\langle B\left(s, X_{s}^{\left(n_{k}\right)}\right), B\left(s, \phi_{s}\right)\right\rangle_{\mathcal{T}_{2}(U, H)} \\
& -2\left(K+\rho\left(\phi_{s}\right)\right)\left\langle X_{s}^{\left(n_{k}\right)}, \phi_{s}\right\rangle_{H}+\left(K+\rho\left(\phi_{s}\right)\right)\left\|\phi_{s}\right\|_{H}^{2} \\
& \left.\left.\left.+\int_{D^{c}}\left(2\left\langle f\left(s, X_{s}^{\left(n_{k}\right)}, z\right), f\left(s, \phi_{s}, z\right)\right\rangle_{H}-\left\|f\left(s, \phi_{s}, z\right)\right\|_{H}^{2}\right) \nu(\mathrm{d} z)\right) \mathrm{d} s\right) \mathrm{~d} t\right] \\
& =\mathbb{E}\left[\int _ { 0 } ^ { T } \psi _ { t } \left(\int _ { 0 } ^ { t } e ^ { - \int _ { 0 } ^ { s } ( K + \rho ( \phi _ { r } ) ) \mathrm { d } r } \left(2_{V^{*}}\left\langle Y_{s}-A\left(s, \phi_{s}\right), \phi_{s}\right\rangle_{V}\right.\right.\right. \\
& +2_{V^{*}}\left\langle A\left(s, \phi_{s}\right), \bar{X}_{s}\right\rangle_{V}-\left\|B\left(s, \phi_{s}\right)\right\|_{2}^{2}+2\left\langle Z_{s}, B\left(s, \phi_{s}\right)\right\rangle_{\mathcal{T}_{2}(U, H)} \\
& -2\left(K+\rho\left(\phi_{s}\right)\right)\left\langle\bar{X}_{s}, \phi_{s}\right\rangle_{H}+\left(K+\rho\left(\phi_{s}\right)\right)\left\|\phi_{s}\right\|_{H}^{2} \\
& \left.\left.\left.+\int_{D^{c}}\left(2\left\langle F(s, z), f\left(s, \phi_{s}, z\right)\right\rangle_{H}-\left\|f\left(s, \phi_{s}, z\right)\right\|_{H}^{2}\right) \nu(\mathrm{d} z)\right) \mathrm{d} s\right) \mathrm{~d} t\right] .
\end{aligned}
$$

On the other hand, by equality (3.18) we have for $\phi \in K \cap \mathcal{M} \cap L^{\infty}\left([0, T] ; L^{p}(\Omega ; H)\right)$,

$$
\begin{aligned}
& \mathbb{E}\left(e^{-\int_{0}^{t}\left(K+\rho\left(\phi_{s}\right)\right) \mathrm{d} s}\left\|X_{t}\right\|_{H}^{2}\right)-\mathbb{E}\left(\left\|X_{0}\right\|_{H}^{2}\right) \\
= & \mathbb{E}\left[\int _ { 0 } ^ { t } e ^ { - \int _ { 0 } ^ { s } ( K + \rho ( \phi _ { r } ) ) \mathrm { d } r } \left(2_{V^{*}}\left\langle Y_{s}, \bar{X}_{s}\right\rangle_{V}+\left\|Z_{s}\right\|_{2}^{2}\right.\right. \\
& \left.\left.-\left(K+\rho\left(\phi_{s}\right)\right)\left\|X_{s}\right\|_{H}^{2}+\int_{D^{c}}\|F(s, z)\|_{H}^{2} \nu(\mathrm{d} z)\right) \mathrm{d} s\right] .
\end{aligned}
$$

Combining (3.20) with (3.19) we have

$$
\mathbb{E}\left[\int _ { 0 } ^ { T } \psi _ { t } \left(\int _ { 0 } ^ { t } e ^ { - \int _ { 0 } ^ { s } ( K + \rho ( \phi _ { r } ) ) \mathrm { d } r } \left(2_{V^{*}}\left\langle Y_{s}-A\left(s, \phi_{s}\right), \bar{X}_{s}-\phi_{s}\right\rangle_{V}\right.\right.\right.
$$




$$
\begin{aligned}
& -\left(K+\rho\left(\phi_{s}\right)\right)\left\|\bar{X}_{s}-\phi_{s}\right\|_{H}^{2}+\left\|B\left(s, \phi_{s}\right)-Z_{s}\right\|_{2}^{2} \\
& \left.\left.\left.+\int_{D^{c}}\left\|f\left(s, \phi_{s}, z\right)-F(s, z)\right\|_{H}^{2} \nu(\mathrm{d} z)\right) \mathrm{d} s\right) \mathrm{~d} t\right] \leq 0 .
\end{aligned}
$$

Therefore, if we put $\phi=\bar{X}$ in (3.21), we can obtain that $Z=B(\cdot, \bar{X})$ in $J$ and $F(\cdot, \cdot)=$ $f\left(\cdot, \bar{X}_{.}, \cdot\right)$ in $\mathcal{M}$.

Note that (3.21) also implies that

$$
\begin{aligned}
\mathbb{E}\left[\int _ { 0 } ^ { T } \psi _ { t } \left(\int_{0}^{t} e^{-\int_{0}^{s}\left(K+\rho\left(\phi_{r}\right)\right) \mathrm{d} r}\right.\right. & \left(2_{V^{*}}\left\langle Y_{s}-A\left(s, \phi_{s}\right), \bar{X}_{s}-\phi_{s}\right\rangle_{V}\right. \\
& \left.\left.\left.-\left(K+\rho\left(\phi_{s}\right)\right)\left\|\bar{X}_{s}-\phi_{s}\right\|_{H}^{2}\right) \mathrm{~d} s\right) \mathrm{~d} t\right] \leq 0 .
\end{aligned}
$$

Put $\phi=\bar{X}-\varepsilon \tilde{\phi} v$ in (3.22) for $\tilde{\phi} \in L^{\infty}([0, T] \times \Omega ; \mathrm{d} t \times \mathbb{P} ; \mathbb{R})$ and $v \in V$, divide both sides by $\varepsilon$ and let $\varepsilon \rightarrow 0$. Then we have

$$
\mathbb{E}\left[\int_{0}^{T} \psi_{t}\left(\int_{0}^{t} e^{-\int_{0}^{s}\left(K+\rho\left(\bar{X}_{r}\right)\right) \mathrm{d} r}\left(2 \tilde{\phi}_{s V^{*}}\left\langle Y_{s}-A\left(s, \bar{X}_{s}\right), v\right\rangle_{V}\right) \mathrm{d} s\right) \mathrm{d} t\right] \leq 0
$$

Hence, we infer $Y=A(\cdot, \bar{X})$.

Therefore, we conclude that the process $X=\left\{X_{t}\right\}_{t \geq 0}$ is a solution to (3.1). Furthermore, the estimates (1.5) and (1.6) can be proved for $\left\{X_{t}\right\}$ by the same arguments in Lemmas 3.2 and 3.3 .

Uniqueness of solutions: we finally proceed to show the uniqueness of solutions to problem (3.1).

Suppose that $X=\left(X_{t}\right)$ and $Y=\left(Y_{t}\right)$ are the solutions of (3.1) with initial conditions $X_{0}, Y_{0}$ respectively, i.e.

$$
\begin{gathered}
X_{t}=X_{0}+\int_{0}^{t} A\left(s, X_{s}\right) \mathrm{d} s+\int_{0}^{t} B\left(s, X_{s}\right) \mathrm{d} W_{s}+\int_{0}^{t} \int_{D^{c}} f\left(s, X_{s-}, z\right) \tilde{N}(\mathrm{~d} s, \mathrm{~d} z), t \in[0, T] \\
Y_{t}=Y_{0}+\int_{0}^{t} A\left(s, Y_{s}\right) \mathrm{d} s+\int_{0}^{t} B\left(s, Y_{s}\right) \mathrm{d} W_{s}+\int_{0}^{t} \int_{D^{c}} f\left(s, Y_{s-}, z\right) \tilde{N}(\mathrm{~d} s, \mathrm{~d} z), t \in[0, T] .
\end{gathered}
$$

We define the following stopping times:

$$
\sigma_{N}:=\inf \left\{t \in[0, T]:\left\|X_{t}\right\|_{H} \geq N\right\} \wedge \inf \left\{t \in[0, T]:\left\|Y_{t}\right\|_{H} \geq N\right\} \wedge T
$$


Applying again the Schmalfuss [54] trick, by means of the Itô formula (3.18) we have

$$
\begin{aligned}
& e^{-\int_{0}^{t \wedge \sigma_{N}}\left(K+\rho\left(Y_{s}\right)\right) \mathrm{d} s}\left\|X_{t \wedge \sigma_{N}}-Y_{t \wedge \sigma_{N}}\right\|_{H}^{2}-\left\|X_{0}-Y_{0}\right\|_{H}^{2} \\
= & \int_{0}^{t \wedge \sigma_{N}} e^{-\int_{0}^{s}\left(K+\rho\left(Y_{r}\right)\right) \mathrm{d} r}\left(2_{V^{*}}\left\langle A\left(s, X_{s}\right)-A\left(s, Y_{s}\right), X_{s}-Y_{s}\right\rangle_{V}\right. \\
& \left.+\left\|B\left(s, X_{s}\right)-B\left(s, Y_{s}\right)\right\|_{2}^{2}-\left(K+\rho\left(Y_{s}\right)\right)\left\|X_{s}-Y_{s}\right\|_{H}^{2}\right) \mathrm{d} s \\
& +2 \int_{0}^{t \wedge \sigma_{N}} e^{-\int_{0}^{s}\left(K+\rho\left(Y_{r}\right)\right) \mathrm{d} r}\left\langle X_{s}-Y_{s}, B\left(s, X_{s}\right) \mathrm{d} W_{s}-B\left(s, Y_{s}\right) \mathrm{d} W_{s}\right\rangle_{H} \\
& +2 \int_{0}^{t \wedge \sigma_{N}} \int_{D^{c}} e^{-\int_{0}^{s}\left(K+\rho\left(Y_{r}\right)\right) \mathrm{d} r}\left\langle X_{s}-Y_{s}, f\left(s, X_{s-}, z\right)-f\left(s, Y_{s-}, z\right)\right\rangle_{H} \tilde{N}(\mathrm{~d} s, \mathrm{~d} z) \\
& +\int_{0}^{t \wedge \sigma_{N}} \int_{D^{c}} e^{-\int_{0}^{s}\left(K+\rho\left(Y_{r}\right)\right) \mathrm{d} r}\left\|f\left(s, X_{s-}, z\right)-f\left(s, Y_{s-}, z\right)\right\|_{H}^{2} N(\mathrm{~d} s, \mathrm{~d} z) .
\end{aligned}
$$

It then follows from assumption $(H 2)$ that

$$
\begin{aligned}
& \mathbb{E}\left[e^{-\int_{0}^{t \wedge \sigma_{N}}\left(K+\rho\left(Y_{s}\right)\right) \mathrm{d} s}\left\|X_{t}-Y_{t}\right\|_{H}^{2}\right]-\mathbb{E}\left\|X_{0}-Y_{0}\right\|_{H}^{2} \\
= & \mathbb{E}\left[\int _ { 0 } ^ { t \wedge \sigma _ { N } } e ^ { - \int _ { 0 } ^ { s } ( K + \rho ( Y _ { r } ) ) \mathrm { d } r } \left(2_{V^{*}}\left\langle A\left(s, X_{s}\right)-A\left(s, Y_{s}\right), X_{s}-Y_{s}\right\rangle_{V}\right.\right. \\
& +\left\|B\left(s, X_{s}\right)-B\left(s, Y_{s}\right)\right\|_{2}^{2}-\left(K+\rho\left(Y_{s}\right)\right)\left\|X_{s}-Y_{s}\right\|_{H}^{2} \\
& \left.\left.+\int_{D^{c}}\left\|f\left(s, X_{s-}, z\right)-f\left(s, Y_{s-}, z\right)\right\|_{H}^{2} \nu(\mathrm{d} z)\right) \mathrm{d} s\right] \leq 0 .
\end{aligned}
$$

Hence if $X_{0}=Y_{0} \mathbb{P}$-a.s., then

$$
\mathbb{E}\left[e^{-\int_{0}^{t \wedge \sigma_{N}}\left(K+\rho\left(Y_{s}\right)\right) \mathrm{d} s}\left\|X_{t}-Y_{t}\right\|_{H}^{2}\right]=0, t \in[0, T] .
$$

Note that by (1.4) and (1.5) (see Lemma 3.2) we have

$$
\int_{0}^{T}\left(K+\rho\left(Y_{s}\right)\right) \mathrm{d} s<\infty, \mathbb{P} \text {-a.s.. }
$$

Therefore, by letting $N \rightarrow \infty$ (hence $\sigma_{N} \uparrow T$ ) we have that $X_{t}=Y_{t}$, $\mathbb{P}$-a.s., $t \in[0, T]$. Then the pathwise uniqueness follows from the path càdlàg property of $X, Y$ in $H$.

This completes the proof of Theorem 3.1.

\section{$3.2 \quad$ With large jumps}

Let $\tau$ be a stopping time such that $\tau<\infty$ a.s.. We define

$$
\begin{aligned}
W^{\tau}(t) & =W(t+\tau)-W(\tau) ; \\
p^{\tau}(t) & =p(t+\tau), t \in \mathcal{D}\left(p^{\tau}\right),
\end{aligned}
$$

where $\mathcal{D}\left(p^{\tau}\right)=\{t \in(0, \infty): t+\tau \in \mathcal{D}(p)\}$. Let $\mathcal{F}_{t}^{\tau}=\mathcal{F}_{t+\tau}, t \in[0, T-\tau]$. The following result is a direct extension of Theorem II6.4 and II6.5 in [31. 
Proposition 3.5. The process $W^{\tau}$ defined by (3.24) is a cylindrical $\mathcal{F}_{t}^{\tau}$-Wiener process and $p^{\tau}$ is a stationary $\mathcal{F}_{t}^{\tau}$-Poisson point process with the intensity measure $\nu$.

Clearly $W^{\tau}$ is independent of $\mathcal{F}_{\tau}$ and $W^{\tau}, p^{\tau}$ enjoy the same properties as $W, p$.

Corollary 3.6. Let $\tau$ be an $[0, T]$-valued stopping time on and $X_{\tau}$ be an $\mathcal{F}_{\tau}$-measurable random variable. Under the assumptions of Theorem 1.2, there exists a unique càdlàg $H$ valued $\mathbb{F}$-adapted process $\left(X_{t}\right)$ and a process $\bar{X} \in L^{\alpha}([\tau, T] ; V) \cap L^{2}([\tau, T] ; H)$, $\mathbb{P}$-a.s. which is $\mathrm{d} t \times \mathbb{P}$-equivalent to $X$ such that the equality holds $\mathbb{P}$-a.s.:

$$
X_{t}=X_{\tau}+\int_{\tau}^{t} A\left(s, \bar{X}_{s}\right) \mathrm{d} s+\int_{\tau}^{t} B\left(s, \bar{X}_{s}\right) \mathrm{d} W_{s}+\int_{\tau}^{t} \int_{D^{c}} f\left(s, \bar{X}_{s-}, z\right) \tilde{N}(\mathrm{~d} s, \mathrm{~d} z), t \in[\tau, T] .
$$

Moreover, if $X_{\tau} \in L^{\beta+2}\left(\Omega, \mathcal{F}_{\tau}, \mathbb{P} ; H\right)$, then we have

$$
\bar{X} \in L^{\alpha}([\tau, T] \times \Omega, \mathrm{d} t \times \mathbb{P} ; V) \cap L^{\beta+2}([\tau, T] \times \Omega, \mathrm{d} t \times \mathbb{P} ; H) .
$$

Proof. We first assume $X_{\tau}=h \in H$, then it is obvious that $X_{\tau} \in L^{\beta+2}\left(\Omega, \mathcal{F}_{\tau}, \mathbb{P} ; H\right)$.

Let $N^{\tau}$ be the compensated Poisson random measure associated to the Poisson point process $p^{\tau}$. As an immediate consequence of Theorem 3.1, there exists a unique $\left(\mathcal{F}_{t}^{\tau}\right)$-adapted $H$-valued càdlàg process $X^{\tau, h}$ such that

$$
\begin{aligned}
X_{t}^{\tau, h}= & +\int_{0}^{t} A\left(s+\tau, \bar{X}_{s}^{\tau, h}\right) d s+\int_{0}^{t} B\left(s+\tau, \bar{X}_{s}^{\tau, h}\right) \mathrm{d} W_{s}^{\tau} \\
& +\int_{0}^{t} \int_{D^{c}} f\left(s+\tau, \bar{X}_{s-}^{\tau, h}, z\right) \tilde{N}^{\tau}(\mathrm{d} s, \mathrm{~d} z), t \in[0, T-\tau],
\end{aligned}
$$

where as before $\bar{X}^{\tau, h}$ is the $\mathrm{d} t \times \mathbb{P}$-equivalent class of $X^{\tau, h}$. Indeed, this follows along the same lines of the proof of Theorem 3.1 in such a way that all computations involving the expectations are replaced by conditional expectations with respect to $\mathcal{F}_{\tau}$.

Since for any $h \in H$, the solution $X_{t}^{\tau, h}$ is a measurable function of $h$, by replacing $h$ with the $\mathcal{F}_{\tau}$-measurable random variable $X_{\tau}$, where $X_{\tau}, W^{\tau}$ and $p^{\tau}$ are mutually independent, we obtain an unique solution $X^{\tau}$ satisfying

$$
\begin{aligned}
X_{t}^{\tau}= & X_{\tau}+\int_{0}^{t} A\left(s+\tau, \bar{X}_{s}^{\tau}\right) d s+\int_{0}^{t} B\left(s+\tau, \bar{X}_{s}^{\tau}\right) \mathrm{d} W_{s}^{\tau} \\
& +\int_{0}^{t} \int_{D^{c}} f\left(s+\tau, \bar{X}_{s-}^{\tau}, z\right) \tilde{N}^{\tau}(\mathrm{d} s, \mathrm{~d} z), t \in[0, T-\tau] .
\end{aligned}
$$

Set $X_{t}:=X_{t-\tau}^{\tau}$ and $\bar{X}_{t}:=\bar{X}_{t-\tau}^{\tau}$, for $t \in[\tau, T]$, then it is straightforward to see that $X$ satisfies Equation (3.25) with the initial condition $X_{\tau}$.

For convenience, we use $X_{\tau, t}^{\prime}(\xi), t \in[\tau, T]$ to denote the solution to Equation (3.25) on $[\tau, T]$ with initial condition $\xi$ at time $\tau$ and $X_{0, t}(x), t \in[0, T]$ to denote the solution to equation (1.1) on $[0, T]$ with initial condition $x$ at time 0 . 
Theorem 3.1 tells us that equation (3.1) with initial condition $x$ at time 0 has a unique $H$-valued càdlàg solution $X^{\prime}:=\left(X_{0, t}^{\prime}(x)\right)_{t \in[0, T]}$ on $[0, T]$, that is

$$
\begin{aligned}
X_{0, t}^{\prime}(x)= & +\int_{0}^{t} A\left(s, \bar{X}_{0, s}^{\prime}(x)\right) \mathrm{d} s+\int_{0}^{t} B\left(s, \bar{X}_{0, s}^{\prime}(x)\right) \mathrm{d} W_{s} \\
& +\int_{0}^{t} \int_{D^{c}} f\left(s, \bar{X}_{0, s-}^{\prime}(x), z\right) \tilde{N}(\mathrm{~d} s, \mathrm{~d} z), t \in[0, T] .
\end{aligned}
$$

Here $\bar{X}_{0, \cdot}^{\prime}(x) \in L^{\alpha}([0, T] \times \Omega, \mathrm{d} t \times \mathbb{P} ; V) \cap L^{2}([0, T] \times \Omega, \mathrm{d} t \times \mathbb{P} ; H)$ and it is $\mathrm{d} t \times \mathbb{P}$-equivalent to $X_{0, \cdot}^{\prime}(x)$. Recall that $\left\{\tau_{n}\right\}$ are the arrival times for the jumps of the Poisson process $N(t, D)$, $t \in[0, T]$. Now we may construct a solution to (1.1) on $\left[0, \tau_{1}\right]$ as follows:

$$
X_{0, t}(x)=\left\{\begin{array}{cc}
X_{0, t}^{\prime}(x), & \text { for } 0 \leq t<\tau_{1} \\
X_{0, \tau_{1}-}^{\prime}(x)+g\left(\tau_{1}, \bar{X}_{0, \tau_{1}-}^{\prime}(x), p\left(\tau_{1}\right)\right), & \text { for } t=\tau_{1}
\end{array}\right.
$$

We note that since the process $X_{0, t}^{\prime}(x), t \in[0, T]$ has no jumps occurring at time $\tau_{1}$, we infer $X_{0, \tau_{1}-}(x)=X_{0, \tau_{1}-}^{\prime}(x)=X_{0, \tau_{1}}^{\prime}(x)$. Set $\bar{X}_{0, t}(x)=\bar{X}_{0, t}^{\prime}(x)$ on $\left[0, \tau_{1}\right)$ and $\bar{X}_{0, \tau_{1}}(x)=$ $\bar{X}_{0, \tau_{1}-}^{\prime}(x)+g\left(\tau_{1}, \bar{X}_{0, \tau_{1}-}^{\prime}(x), p\left(\tau_{1}\right)\right)$. It clearly forces that $\bar{X}_{0, t}(x)$ is $\mathrm{d} t \times \mathbb{P}$-equivalent to $X_{0, t}(x)$ on $\left[0, \tau_{1}\right]$. Hence we have

$$
\begin{aligned}
X_{0, \tau_{1}}(x)= & X_{0, \tau_{1}-}^{\prime}(x)+g\left(\tau_{1}, \bar{X}_{0, \tau_{1}-}^{\prime}(x), p\left(\tau_{1}\right)\right) \\
=x & +\int_{0}^{\tau_{1}} A\left(s, \bar{X}_{0, s}(x)\right) \mathrm{d} s+\int_{0}^{\tau_{1}} B\left(s, \bar{X}_{0, s}(x)\right) \mathrm{d} W_{s} \\
& +\int_{0}^{\tau_{1}} \int_{D^{c}} f\left(s, \bar{X}_{0, s-}(x), z\right) \tilde{N}(\mathrm{~d} s, \mathrm{~d} z)+g\left(\tau_{1}, \bar{X}_{0, \tau_{1}-}(x), p\left(\tau_{1}\right)\right) .
\end{aligned}
$$

Also, since $\tau_{1}$ is the first jump time of the process $N(t, D), t \in[0, T]$, we infer that

$$
\int_{0}^{t} \int_{D} g\left(s, \bar{X}_{0, s-}^{\prime}(x), z\right) N(\mathrm{~d} s, \mathrm{~d} z)=\left\{\begin{array}{cc}
0, & t \in\left[0, \tau_{1}\right) \\
g\left(\tau_{1}, \bar{X}_{0, \tau_{1}-}^{\prime}(x), p\left(\tau_{1}\right)\right), & t \in\left[\tau_{1}, \tau_{2}\right) .
\end{array}\right.
$$

It follows that for $t \in\left[0, \tau_{1}\right]$ we have

$$
\begin{aligned}
X_{0, t}(x)= & +\int_{0}^{t} A\left(s, \bar{X}_{0, s}(x)\right) \mathrm{d} s+\int_{0}^{t} B\left(s, \bar{X}_{0, s}(x)\right) \mathrm{d} W_{s} \\
& +\int_{0}^{t} \int_{D^{c}} f\left(s, \bar{X}_{0, s-}(x), z\right) \tilde{N}(\mathrm{~d} s, \mathrm{~d} z)+\int_{0}^{t} \int_{D} g\left(s, \bar{X}_{0, s-}(x), z\right) N(\mathrm{~d} s, \mathrm{~d} z),
\end{aligned}
$$

which shows that the process $X_{0, t}(x)$ is an $H$-valued solution to the equation (1.1) on [0, $\left.\tau_{1}\right]$.

Since the valued of $g(\cdot, X, \cdot)$ at time $\tau_{1}$ depends only on the valued of $X_{\tau_{1}-}$ strictly prior to the time $\tau_{1}$, the uniqueness of the solution $X_{0, t}^{\prime}(x)$ on $\left[0, \tau_{1}\right)$ implies the uniqueness of the solution $X_{0, t}(x)$ on $\left[0, \tau_{1}\right]$.

According to Corollary 3.6, let us denote $X_{\tau_{1}, t}^{\prime}\left(X_{0, \tau_{1}}(x)\right)$ the unique solution to the equation (3.1) with initial condition $X_{0, \tau_{1}}(x)$ at time $\tau_{1}$, then there exists a $\mathrm{d} t \times \mathbb{P}$-equivalent 
class $\bar{X}_{\tau_{1}, t}^{\prime}\left(X_{0, \tau_{1}}(x)\right), t \in\left[\tau_{1}, T\right]$ satisfying

$$
\begin{aligned}
X_{\tau_{1}, t}^{\prime}\left(X_{0, \tau_{1}}(x)\right)=X_{0, \tau_{1}}(x) & +\int_{\tau_{1}}^{t} A\left(s, \bar{X}_{\tau_{1}, s}^{\prime}\left(X_{0, \tau_{1}}(x)\right)\right) \mathrm{d} s+\int_{\tau_{1}}^{t} B\left(s, \bar{X}_{\tau_{1}, s}^{\prime}\left(X_{0, \tau_{1}}(x)\right)\right) \mathrm{d} W_{s} \\
& +\int_{\tau_{1}}^{t} \int_{D^{c}} f\left(s, \bar{X}_{\tau_{1}, s-}^{\prime}\left(X_{0, \tau_{1}}(x)\right), z\right) \tilde{N}(\mathrm{~d} s, \mathrm{~d} z), \quad t \in\left[\tau_{1}, T\right] .
\end{aligned}
$$

We define

$$
X_{0, t}(x)=\left\{\begin{array}{cc}
X_{0, t}(x), & \text { for } 0 \leq t \leq \tau_{1} \\
X_{\tau_{1}, t}^{\prime}\left(X_{0, \tau_{1}}(x)\right), & \text { for } \tau_{1}<t<\tau_{2} \\
X_{\tau_{1}, \tau_{2}-}^{\prime}\left(X_{0, \tau_{1}}(x)\right)+g\left(\tau_{2}, \bar{X}_{\tau_{1}, \tau_{2}-}^{\prime}\left(X_{0, \tau_{1}}(x)\right), p\left(\tau_{2}\right)\right), & \text { for } t=\tau_{2}
\end{array}\right.
$$

and

$$
\bar{X}_{0, t}(x)=\left\{\begin{array}{cc}
\bar{X}_{0, t}(x), & \text { for } 0 \leq t \leq \tau_{1} ; ; \\
\bar{X}_{\tau_{1}, t}^{\prime}\left(X_{0, \tau_{1}}(x)\right), & \text { for } \tau_{1}<t<\tau_{2} \\
\bar{X}_{\tau_{1}, \tau_{2}-}^{\prime}\left(X_{0, \tau_{1}}(x)\right)+g\left(\tau_{2}, \bar{X}_{\tau_{1}, \tau_{2}-}^{\prime}\left(X_{0, \tau_{1}}(x)\right), p\left(\tau_{2}\right)\right), & \text { for } t=\tau_{2},
\end{array}\right.
$$

Clearly, $\bar{X}_{0, s}(x)=X_{0, s}(x), \mathrm{d} t \times \mathbb{P}$ on $\left[0, \tau_{2}\right]$. Then we have for $t \in\left(\tau_{1}, \tau_{2}\right)$,

$$
\begin{aligned}
X_{0, t}(x)= & X_{\tau_{1}, t}^{\prime}\left(X_{0, \tau_{1}}(x)\right) \\
= & x+\int_{0}^{t} A\left(s, \bar{X}_{0, s}(x)\right) \mathrm{d} s+\int_{0}^{t} B\left(s, \bar{X}_{0, s}^{\prime}(x)\right) \mathrm{d} W_{s} \\
& +\int_{0}^{t} \int_{D^{c}} f\left(s, \bar{X}_{0, s-}(x), z\right) \tilde{N}(\mathrm{~d} s, \mathrm{~d} z)+\int_{0}^{t} \int_{D} g\left(s, \bar{X}_{0, s-}(x), z\right) N(\mathrm{~d} s, \mathrm{~d} z) .
\end{aligned}
$$

As we known that $X_{\tau_{1}, \tau_{2}-}^{\prime}\left(X_{0, \tau_{1}}(x)\right)=X_{\tau_{1}, \tau_{2}}^{\prime}\left(X_{0, \tau_{1}}(x)\right)$, a similar argument as above gives

$$
\begin{aligned}
X_{0, \tau_{2}}(x)= & x+\int_{0}^{\tau_{2}} A\left(s, \bar{X}_{0, s}(x)\right) \mathrm{d} s+\int_{0}^{\tau_{2}} B\left(s, \bar{X}_{0, s}(x)\right) \mathrm{d} W_{s} \\
& +\int_{0}^{\tau_{2}} \int_{D^{c}} f\left(s, \bar{X}_{0, s-}(x), z\right) \tilde{N}(\mathrm{~d} s, \mathrm{~d} z)+\int_{0}^{\tau_{2}} \int_{D} g\left(s, \bar{X}_{0, s-}(x), z\right) N(\mathrm{~d} s, \mathrm{~d} z) .
\end{aligned}
$$

In particular,

$$
\begin{aligned}
\int_{0}^{\tau_{2}} \int_{D} g\left(s, \bar{X}_{0, s-}(x), z\right) N(\mathrm{~d} s, \mathrm{~d} z) & =g\left(\tau_{1}, \bar{X}_{0, \tau_{1}-}, p\left(\tau_{1}\right)\right)+g\left(\tau_{2}, \bar{X}_{0, \tau_{2}-}, p\left(\tau_{2}\right)\right) \\
& =g\left(\tau_{1}, \bar{X}_{0, \tau_{1}-}^{\prime}(x), p\left(\tau_{1}\right)\right)+g\left(\tau_{2}, \bar{X}_{\tau_{1}, \tau_{2}-}^{\prime}\left(X_{0, \tau_{1}}(x)\right), p\left(\tau_{2}\right)\right) .
\end{aligned}
$$

Therefore, $X_{0, t}(x)$ is a solution of (1.1) on $\left[0, \tau_{2}\right]$ and the uniqueness of the solution on $\left[0, \tau_{2}\right]$ follows from the uniqueness of the solutions $X_{0, t}^{\prime}(x)$ and $X_{\tau_{1}, t}^{\prime}\left(X_{0, \tau_{1}}\right)(x)$.

By using this type of interlacing structure, one can construct a unique solution recursively to the equation (1.1) in the time interval $\left[0, \tau_{n}\right]$ for every $n \in \mathbb{N}$.

Now the proof of Corollary 3.6 is complete. 


\section{Application and Examples}

Theorem 1.2 gives a unified framework for a very large class of SPDE driven by general Lévy noise, which generalizes both the classical results in [29, 46, 48] and the recent results in [13, 36]. Within this framework, the issue of the existence and uniqueness of solutions to a large class of stochastic evolution equations with monotone coefficients (cf. [48, 29] for the stochastic porous medium equation and stochastic $p$-Laplace equation) and with locally monotone coefficients (cf. [36, 13] for stochastic Burgers type equations, stochastic 2D Navier-Stokes equations and many other stochastic hydrodynamical systems) driven by more general Lévy processes instead of Wiener processes can be treated.

For the simplicity of notation we use $D_{i}$ to denote the spatial derivative $\frac{\partial}{\partial x_{i}}$, and $\Lambda \subseteq \mathbb{R}^{d}$ is an open bounded domain with smooth boundary. For the standard Sobolev space $W_{0}^{1, p}(\Lambda)$ $(p \geq 2)$ we always use the following (equivalent) Sobolev norm:

$$
\|u\|_{1, p}:=\left(\int_{\Lambda}|\nabla u(x)|^{p} d x\right)^{1 / p} .
$$

For $d=2$, we recall the following well-known estimate on $\mathbb{R}^{2}$ (cf. [56]):

$$
\|u\|_{L^{4}}^{4} \leq C\|u\|_{L^{2}}^{2}\|\nabla u\|_{L^{2}}^{2}, u \in W_{0}^{1,2}(\Lambda) .
$$

We also recall the following estimate on $\mathbb{R}^{3}$ (cf. [56, 41]):

$$
\|u\|_{L^{4}}^{4} \leq C\|u\|_{L^{2}}\|\nabla u\|_{L^{2}}^{3}, u \in W_{0}^{1,2}(\Lambda),
$$

We first recall the following lemma in [50], which partially generalizes the result in [36, Lemma 3.1].

Lemma 4.1. Consider the Gelfand triple

$$
V:=W_{0}^{1,2}(\Lambda) \subset H:=L^{2}(\Lambda) \subset\left(W_{0}^{1,2}(\Lambda)\right)^{*}=V^{*} .
$$

and the operator

$$
A(u)=\Delta u+\langle f(u), \nabla u\rangle,
$$

where $f=\left(f_{1}, \ldots, f_{d}\right): \mathbb{R}^{d} \rightarrow \mathbb{R}^{d}$ is a Lipschitz function and $\langle$,$\rangle denotes inner product in$ $\mathbb{R}^{d}$. Let Lip $(f)$ denote the corresponding Lipschitz constant.

(1) If $d \leq 4$, there exists $C \in] 0, \infty[$ such that for all $u, v, w \in V$

$$
\int_{\Lambda}|u|\left|\nabla w\|v \mid \mathrm{d} x \leq C\| u\left\|_{V}\right\| w\left\|_{V}\right\| v \|_{V}\right.
$$

In particular $A: V \rightarrow V^{*}$ is well defined. Furthermore, if $d=1$ or $f$ is bounded, $A$ satisfies (H4) with $\alpha=2$ and $\beta=2$ or $\beta=0$, respectively.

(2) If $d=1$ or if each $f_{i}$ is bounded and $d=2$, then there exists $C \in(0, \infty)$ such that

$$
2_{V^{*}}\langle A(u)-A(v), u-v\rangle_{V} \leq-\|u-v\|_{V}^{2}+\left(C+C\|v\|_{V}^{2}\right)\|u-v\|_{H}^{2}, u, v \in V .
$$


(3) If $f_{i}$ are bounded and independent of $u$ for $i=1, \cdots, d$, i.e.

$$
A(u)=\Delta u+\langle f, \nabla u\rangle,
$$

then for any $d \geq 1$ we have

$$
2_{V^{*}}\langle A(u)-A(v), u-v\rangle_{V} \leq-\|u-v\|_{V}^{2}+K\|u-v\|_{H}^{2}, u, v \in V .
$$

Proof. The proof can be found in [50], we include it here for the reader's convenience.

(1): We have for all $u, v \in V$

$$
\int_{\Lambda}|\langle f(u), \nabla u\rangle||v| \mathrm{d} x \leq \int_{\Lambda}(|f(0)|+\operatorname{Lip}(f)|u|)|\nabla u||v| \mathrm{d} x .
$$

To prove the first assertion, we note that for all $u, v, w \in V$

$$
\int_{\Lambda}|u||\nabla w||v| \mathrm{d} x \leq\|u v\|_{L^{2}}\|w\|_{V},
$$

and by the generalized Hölder inequality the right hand side is dominated by

(a) $\|u\|_{L^{2}}\|v\|_{L^{\infty}}\|w\|_{V}$,

(b) $\|u\|_{L^{4}}\|v\|_{L^{4}}\|w\|_{V}$,

(c) $\|u\|_{L^{d}}\|v\|_{L^{\frac{2 d}{d-2}}}\|w\|_{V}$.

In the case $d=1, W_{0}^{1,2}(\Lambda) \subset L^{\infty}(\Lambda)$ continuously. Hence assertion (1) follows from (a) if $d=1$.

In the case $d=2, W_{0}^{1,2}(\Lambda) \subset L^{p}(\Lambda)$ continuously for all $p \in[1, \infty[$. Hence assertion (1) follows from (b) if $d=2$.

In the case $d \leq 3, W_{0}^{1,2}(\Lambda) \subset L^{\frac{2 d}{d-2}}(\Lambda)$ continuously, and $L^{\frac{2 d}{d-2}}(\Lambda) \subset L^{d}(\Lambda)$ continuously if $d \leq 4$. Hence assertion (1) follows from (c) if $d=3$ or 4 .

To prove the last part of the assertion we note that this is trivially true if $f$ is bounded. If $d=1$ and if $f$ is merely Lipschitz continuous it follows immediately from (a).

(2): We have

$$
\begin{aligned}
& V^{*}\langle A(u)-A(v), u-v\rangle_{V} \\
= & -\|u-v\|_{V}^{2}+\sum_{i=1}^{d} \int_{\Lambda}\left(f_{i}(u) D_{i} u-f_{i}(v) D_{i} v\right)(u-v) \mathrm{d} x .
\end{aligned}
$$

To estimate the second term on the right hand side, let $F_{i}: \mathbb{R} \rightarrow \mathbb{R}$ be such that $F_{i}(0)=0$ and $F_{i}^{\prime}=f_{i}$ and $G_{i}: \mathbb{R} \rightarrow \mathbb{R}$ be such that $G_{i}(0)=0$ and $G_{i}^{\prime}=F_{i}$. 
Then

$$
\begin{aligned}
& \int_{\Lambda}\left(f_{i}(u) D_{i} u-f_{i}(v) D_{i} v\right)(u-v) \mathrm{d} x \\
= & \int_{\Lambda}\left(f_{i}(u) D_{i}(u-v)+\left(f_{i}(u)-f_{i}(v)\right) D_{i} v\right)(u-v) \mathrm{d} x \\
- & \int_{\Lambda} f_{i}(u-v) D_{i}(u-v)(u-v) \mathrm{d} x \\
+ & \int_{\Lambda} D_{i}\left(F_{i}(u-v)\right)(u-v) \mathrm{d} x
\end{aligned}
$$

where integrating by parts and using that $u-v \in W_{0}^{1,2}(\Lambda)$ we see that the last term on the right hand side is equal to

$$
-\int_{\Lambda} D_{i}\left(G_{i}(u-v)\right) \mathrm{d} x,
$$

which in turn after summation from $i=1$ to $d$ by Gauss's divergence theorem is zero, since $G_{i}(u-v)=0$ on $\partial \Lambda$ for all $1 \leq i \leq d$, because $u, v \in W_{0}^{1,2}(\Lambda)$.

Hence altogether we obtain

$$
\begin{aligned}
& V^{*}\langle A(u)-A(v), u-v\rangle_{V} \\
\leq & -\|u-v\|_{V}^{2}+\int_{\Lambda}\langle f(u)-f(u-v), \nabla(u-v)\rangle(u-v) d x \\
& +\int_{\Lambda}\langle f(u)-f(v), \nabla v\rangle(u-v) \mathrm{d} x
\end{aligned}
$$

Now let us first consider the case $d=1$. Then using that $f$ is Lipschitz and applying Cauchy-Schwarz's and Young's inequalities we estimate, the right hand side of (4.3) by

$$
\begin{aligned}
& -\|u-v\|_{V}^{2}+\operatorname{Lip}(f)\left(\|u-v\|_{V}\|v\|_{L^{\infty}}\|u-v\|_{L^{2}}+\|v\|_{V}\|u-v\|_{L^{4}}^{2}\right) \\
\leq- & -\frac{3}{4}\|u-v\|_{V}^{2}+C\left(\|v\|_{V}^{2}\|u-v\|_{L^{2}}^{2}+\|v\|_{V}\|u-v\|_{L^{4}}^{2}\right)
\end{aligned}
$$

where $C \in(0, \infty)$ is independent of $u, v$ and we used that $W_{0}^{1,2}(\Lambda) \subset L^{\infty}(\Lambda)$ continuously, since $d=1$.

In the case $d=2$ and $f$ is bounded, we similarly obtain that the right hand side of (4.3) is dominated by

$$
\begin{aligned}
& -\|u-v\|_{V}^{2}+2\|f\|_{L^{\infty}}\|u-v\|_{V}\|u-v\|_{L^{2}}+\operatorname{Lip}(f)\|v\|_{V}\|u-v\|_{L^{4}}^{2} \\
\leq- & \frac{3}{4}\|u-v\|_{V}^{2}+C\left(\|u-v\|_{L^{2}}^{2}+\|v\|_{V}\|u-v\|_{L^{4}}^{2}\right),
\end{aligned}
$$

where $C \in(0, \infty)$ is independent of $u, v$.

Hence combining (4.1) with (4.4), (4.5) and using Young's inequality we deduce that for some $C \in(0, \infty)$

$$
V^{*}\langle A(u)-A(v), u-v\rangle_{V} \leq-\frac{1}{2}\|u-v\|_{V}^{2}+\left(C+C\|v\|_{V}^{2}\right)\|u-v\|_{H}^{2} \text { for all } u, v \in V
$$


and assertion (2) is proved.

(3) : In this case $A$ is a linear operator and the assertion follows easily by the similar argument as in (2) (cf. also [36]).

For all examples presented in the remainder of this section, we will only state the result on the existence and uniqueness of solutions. But we should remark that one can also obtain those regularity estimates (1.5) and (1.6) by Theorem 1.2 if we do not have the large jumps term in our equations (i.e. $g=0$ ).

\subsection{Semilinear type SPDEs}

Example 4.2. (Stochastic multidimensional Burgers type equations) Let $\Lambda$ be an open bounded domain in $\mathbb{R}^{d}$ with smooth boundary. We consider the following semilinear stochastic equation

$$
\begin{aligned}
\mathrm{d} X_{t}= & \left(\Delta X_{t}+\left\langle f\left(X_{t}\right), \nabla X_{t}\right\rangle+f_{0}\left(X_{t}\right)\right) \mathrm{d} t+B\left(X_{t}\right) \mathrm{d} W_{t} \\
& +\int_{D^{c}} h\left(X_{t-}, z\right) \tilde{N}(\mathrm{~d} t, \mathrm{~d} z)+\int_{D} g\left(X_{t-}, z\right) N(\mathrm{~d} t, \mathrm{~d} z) ; \\
X_{0}= & x .
\end{aligned}
$$

Suppose the coefficients satisfy the following conditions:

(i) $f=\left(f_{1}, \cdots, f_{d}\right): \mathbb{R} \rightarrow \mathbb{R}^{d}$ is a Lipschitz function;

(ii) $f_{0}$ is a continuous function on $\mathbb{R}$ such that

$$
\begin{aligned}
\left|f_{0}(x)\right| & \leq C\left(|x|^{r}+1\right), x \in \mathbb{R} \\
\left(f_{0}(x)-f_{0}(y)\right)(x-y) & \leq C\left(1+|y|^{s}\right)(x-y)^{2}, x, y \in \mathbb{R} .
\end{aligned}
$$

where $C, r, s$ are some positive constants;

(iii) the function $B: W_{0}^{1,2}(\Lambda) \rightarrow \mathcal{T}_{2}\left(U ; L^{2}(\Lambda)\right)$ satisfies the following Lipschitz condition:

$$
\left\|B\left(v_{1}\right)-B\left(v_{2}\right)\right\|_{2}^{2} \leq C \int_{\Lambda}\left|v_{1}-v_{2}\right|^{2} \mathrm{~d} x, v_{1}, v_{2} \in W_{0}^{1,2}(\Lambda) .
$$

(iv) $h, g: \mathbb{R} \times Z \rightarrow \mathbb{R}$ such that for all $v, v_{1}, v_{2} \in W_{0}^{1,2}(\Lambda)$,

$$
\begin{aligned}
& \int_{D^{c}} \int_{\Lambda}\left|h\left(v_{1}, z\right)-h\left(v_{2}, z\right)\right|^{2} \mathrm{~d} x \nu(\mathrm{d} z) \leq C \int_{\Lambda}\left|v_{1}-v_{2}\right|^{2} \mathrm{~d} x \\
& \int_{D^{c}} \int_{\Lambda}|h(v, z)|^{2} \mathrm{~d} x \nu(\mathrm{d} z) \leq C\left(1+\int_{\Lambda}|v|^{2} \mathrm{~d} x\right) \\
& \int_{D^{c}}\left(\int_{\Lambda}|h(v, z)|^{2} \mathrm{~d} x\right)^{3} \nu(\mathrm{d} z) \leq C\left(1+\left(\int_{\Lambda}|v|^{2} \mathrm{~d} x\right)^{3}\right) .
\end{aligned}
$$

Then we have the following result: 
(1) If $d=1, r=3, s=2$, then for any $x \in L^{6}\left(\Omega, \mathcal{F}_{0}, \mathbb{P} ; H\right)$, (4.6) has a unique solution $\left\{X_{t}\right\}_{t \in[0, T]}$.

(2) If $d=2, r=\frac{7}{3}, s=2$ and each $f_{i}$ is bounded, then for any $x \in L^{6}\left(\Omega, \mathcal{F}_{0}, \mathbb{P} ; H\right)$, (4.6) has a unique solution $\left\{X_{t}\right\}_{t \in[0, T]}$.

(3) If $d=3, r=\frac{7}{3}, s=\frac{4}{3}$ and each $f_{i}$ is bounded measurable function which is independent of $X_{t}$, then for any $x \in L^{6}\left(\Omega, \mathcal{F}_{0}, \mathbb{P} ; H\right)$, (4.6) has a unique solution $\left\{X_{t}\right\}_{t \in[0, T]}$.

Proof. We consider the following Gelfand triple

$$
V:=W_{0}^{1,2}(\Lambda) \subseteq H:=L^{2}(\Lambda) \subseteq\left(W_{0}^{1,2}(\Lambda)\right)^{*}=V^{*}
$$

and define the operator

$$
A(u)=\Delta u+\langle f(u), \nabla u\rangle+f_{0}(u), u \in V
$$

By Lemma 4.1, one can show that $A, B$ satisfies $(H 1)-(H 4)$ with $\alpha=2, \beta=4$ (see [36, Example 3.2]).

Moreover, it is easy to show that $h$ also satisfies the required conditions (i.e. (H2), (1.2) and $(1.3)$ ) by (4.8) .

Then all assertions follow from Theorem 1.2 .

Remark 4.3. (1) If $d=1$ and $f(x)=x$, Theorem 1.2 can be applied to classical stochastic Burgers equation (i.e. (4.6) with $f_{0} \equiv 0$ ). Therefore, the above example improves the main result in [16] (Theorem 2.2) in the sense that we allow the coefficient $B$ in front of Wiener noise to be non-additive type. Another improvement is that we also allow a polynomial perturbation term $f_{0}$ in the drift of (4.6). For example, one can take $f_{0}(x)=$ $-x^{3}+c_{1} x^{2}+c_{2} x\left(c_{1}, c_{2} \in \mathbb{R}\right)$ and show that (4.7) holds. Hence (4.6) also covers some stochastic reaction-diffusion type equations driven by certain type of a Lévy noise (cf. [9]).

(2) If $Z=\mathbb{R}^{d}, D^{c}=\left\{z \in \mathbb{R}^{d}:|z| \leq 1\right\}$ and $\nu$ is a Lévy measure on $\mathbb{R}^{d}$, then one simple sufficient condition for $h$ satisfying (4.8) is to assume

$$
\begin{aligned}
& |h(x, z)-h(y, z)| \leq C|x-y||z|, x, y \in \mathbb{R}, z \in D^{c} \\
& |h(x, z)| \leq C(1+|x|)|z|, x, y \in \mathbb{R}, z \in D^{c}
\end{aligned}
$$

(3) One should note that in the Example 4.2, $B$ is assumed to be Lipschitz from $W_{0}^{1,2}(\Lambda)$ (w.r.t. $\left.\|\cdot\|_{H}\right)$ to $\mathcal{T}_{2}\left(U ; L^{2}(\Lambda)\right)$ only for simplicity. Actually, the Lipschitz condition on $B$ can even be weakened to the requirement

$$
\left\|B\left(v_{1}\right)-B\left(v_{2}\right)\right\|_{2}^{2} \leq\left\|v_{1}-v_{2}\right\|_{V}^{2}+\left(K+K\left\|v_{2}\right\|_{V}^{2}\right)\left\|v_{1}-v_{2}\right\|_{H}^{2}
$$

\subsection{Quasi-linear type SPDEs}

Besides from the example of semilinear SPDE above, we can also apply the main result to the following quasi-linear SPDE on $\mathbb{R}^{d}(d \geq 3)$ driven by Lévy noise. 
Example 4.4. (Stochastic p-Laplace equations) We consider the following equation on $\mathbb{R}^{d}$ for $p>2$

$$
\begin{aligned}
\mathrm{d} X_{t}= & \left(\sum_{i=1}^{d} D_{i}\left(\left|D_{i} X_{t}\right|^{p-2} D_{i} X_{t}\right)+f_{0}\left(X_{t}\right)\right) \mathrm{d} t+B\left(X_{t}\right) \mathrm{d} W_{t} \\
& +\int_{D^{c}} f\left(X_{t-}, z\right) \tilde{N}(\mathrm{~d} t, \mathrm{~d} z)+\int_{D} g\left(X_{t-}, z\right) N(\mathrm{~d} t, \mathrm{~d} z) ; \\
X_{0}= & x
\end{aligned}
$$

Suppose the following conditions hold:

(i) $f_{0}$ is a continuous function on $\mathbb{R}$ such that

$$
\begin{aligned}
f_{0}(x) x & \leq C\left(|x|^{\frac{p}{2}+1}+1\right), x \in \mathbb{R} \\
\left|f_{0}(x)\right| & \leq C\left(|x|^{r}+1\right), x \in \mathbb{R} ; \\
\left(f_{0}(x)-f_{0}(y)\right)(x-y) & \leq C\left(1+|y|^{t}\right)|x-y|^{s}, x, y \in \mathbb{R},
\end{aligned}
$$

where $C>0$ and $r, s, t \geq 1$ are some constants.

(ii) $B: W_{0}^{1, p}(\Lambda) \rightarrow \overline{\mathcal{T}}_{2}\left(U ; L^{2}(\Lambda)\right)$ satisfies the following condition:

$$
\left\|B\left(v_{1}\right)-B\left(v_{2}\right)\right\|_{2}^{2} \leq C \int_{\Lambda}\left|v_{1}-v_{2}\right|^{2} \mathrm{~d} x, v_{1}, v_{2} \in W_{0}^{1, p}(\Lambda) .
$$

(iv) $f, g: \mathbb{R} \times Z \rightarrow \mathbb{R}$ such that for all $v, v_{1}, v_{2} \in W_{0}^{1, p}(\Lambda)$,

$$
\begin{aligned}
& \int_{D^{c}} \int_{\Lambda}\left|f\left(v_{1}, z\right)-f\left(v_{2}, z\right)\right|^{2} \mathrm{~d} x \nu(\mathrm{d} z) \leq C \int_{\Lambda}\left|v_{1}-v_{2}\right|^{2} \mathrm{~d} x \\
& \int_{D^{c}} \int_{\Lambda}|f(v, z)|^{2} \mathrm{~d} x \nu(\mathrm{d} z) \leq C\left(1+\int_{\Lambda}|v|^{2} \mathrm{~d} x\right) \\
& \int_{D^{c}}\left(\int_{\Lambda}|f(v, z)|^{2} \mathrm{~d} x\right)^{3} \nu(\mathrm{d} z) \leq C\left(1+\left(\int_{\Lambda}|v|^{2} \mathrm{~d} x\right)^{3}\right) .
\end{aligned}
$$

Then we have

(1) if $d<p, s=2, r=p+1$ and $t \leq p$, then for any $x \in L^{6}\left(\Omega, \mathcal{F}_{0}, \mathbb{P} ; H\right)$, (4.9) has a unique solution.

(2) if $d>p, 2<s<p, r=\frac{2 p}{d}+p-1$ and $t \leq \min \left\{\frac{p^{2}(s-2)}{(d-p)(p-2)}, \frac{p(p-s)}{p-2}\right\}$, for any $x \in L^{6}\left(\Omega, \mathcal{F}_{0}, \mathbb{P} ; H\right)(4.9)$ has a unique solution.

Proof. (1) We consider the following Gelfand triple $\left(q:=\frac{p}{p-1}\right)$

$$
V:=W_{0}^{1, p}(\Lambda) \subseteq H:=L^{2}(\Lambda) \subseteq W^{-1, q}(\Lambda)=V^{*}
$$

It is well known that $\sum_{i=1}^{d} D_{i}\left(\left|D_{i} u\right|^{p-2} D_{i} u\right)$ satisfy $(H 1)-(H 4)$ with $\alpha=p$ (cf. [34]). In particular, there exists a constant $\delta>0$ such that

$$
\sum_{i=1}^{d} V^{*}\left\langle D_{i}\left(\left|D_{i} u\right|^{p-2} D_{i} u\right)-D_{i}\left(\left|D_{i} v\right|^{p-2} D_{i} v\right), u-v\right\rangle_{V} \leq-\delta\|u-v\|_{V}^{p}, u, v \in W_{0}^{1, p}(\Lambda)
$$


Recall that for $d<p$ we have the following Sobolev embedding

$$
W_{0}^{1, p}(\Lambda) \subseteq L^{\infty}(\Lambda)
$$

Hence by (4.10) we have

$$
\begin{aligned}
V^{*}\left\langle f_{0}(u)-f_{0}(v), u-v\right\rangle_{V} & \leq C \int_{\Lambda}\left(1+|v|^{t}\right)|u-v|^{2} d x \\
& \leq C\left(1+\|v\|_{L^{\infty}}^{t}\right)\|u-v\|_{L^{2}}^{2} \\
& \leq C\left(1+\|v\|_{V}^{t}\right)\|u-v\|_{H}^{2}, u, v \in V,
\end{aligned}
$$

where $C$ is a constant may change from line to line.

Hence (H2) holds with $\rho(v)=C\|v\|_{V}^{t}$.

Note that from (4.10) we have

$$
\begin{aligned}
V^{*}\left\langle f_{0}(u), u\right\rangle_{V} & \leq C \int_{\Lambda}\left(1+|u|^{\frac{p}{2}+1}\right) d x \\
& \leq C\left(1+\|u\|_{L^{\infty}}^{p / 2}\|u\|_{H}\right) \\
& \leq \frac{\delta}{2}\|u\|_{V}^{p}+C\left(1+\|u\|_{H}^{2}\right), u \in V .
\end{aligned}
$$

Therefore, (4.14) together with (4.12) verify (H3) with $\alpha=p$.

(H4) with $\beta=4$ (in fact one may take $\beta=\frac{2 p}{p-1}<4$ ) follows from the following estimate:

$$
\left\|f_{0}(u)\right\|_{V^{*}} \leq C\left(1+\|u\|_{L^{p+1}}^{p+1}\right) \leq C\left(1+\|u\|_{L^{\infty}}^{p-1}\|u\|_{H}^{2}\right) \leq C\left(1+\|u\|_{V}^{p-1}\|u\|_{H}^{2}\right), u \in V .
$$

Then combining with (4.11) we know that the assertions follow from Theorem 1.2 .

(2) Note that for $d>p$ we have the following Sobolev embedding

$$
W_{0}^{1, p}(\Lambda) \subseteq L^{p_{0}}(\Lambda), p_{0}=\frac{d p}{d-p} .
$$

Let $t_{0}=\frac{p(s-2)}{s(p-2)} \in(0,1)$ and $p_{1} \in\left(2, p_{0}\right)$ such that

$$
\frac{1}{p_{1}}=\frac{1-t_{0}}{2}+\frac{t_{0}}{p_{0}}
$$

Then we have the following interpolation inequality:

$$
\|u\|_{L^{p_{1}}} \leq\|u\|_{L^{2}}^{1-t_{0}}\|u\|_{L^{p_{0}}}^{t_{0}}, u \in W_{0}^{1, p}(\Lambda) .
$$

Since $2<s<p$, it is easy to show that $s<p_{1}$.

Let $p_{2}=\frac{p_{1}}{p_{1}-s}$, then by (4.10) we have

$$
\begin{aligned}
V^{*}\left\langle f_{0}(u)-f_{0}(v), u-v\right\rangle_{V} & \leq C \int_{\Lambda}\left(1+|v|^{t}\right)|u-v|^{s} d x \\
& \leq C\left(1+\|v\|_{L^{t p_{2}}}^{t}\right)\|u-v\|_{L^{p_{1}}}^{s} \\
& \leq C\left(1+\|v\|_{L^{t p_{2}}}^{t}\right)\|u-v\|_{L^{2}}^{s\left(-t_{0}\right)}\|u-v\|_{L^{p_{0}}}^{s t_{0}} \\
& \leq \varepsilon\|u-v\|_{L^{p_{0}}}^{p}+C_{\varepsilon}\left(1+\|v\|_{L^{t p_{2}}}^{t b}\right)\|u-v\|_{L^{2}}^{2},
\end{aligned}
$$


where $\varepsilon, C_{\varepsilon}$ are some constants and the last step follows from the following Young inequality

$$
x y \leq \varepsilon x^{a}+C_{\varepsilon} y^{b}, x, y \in \mathbb{R}, a=\frac{p-2}{s-2}, b=\frac{p-2}{p-s} .
$$

With some calculations, one have

$$
\frac{s}{p_{1}}=\frac{p-s}{p-2}+\frac{p(s-2)}{p_{0}(p-2)}, p_{2}=\frac{p_{0}(p-2)}{\left(p_{0}-p\right)(s-2)} .
$$

Hence if $t \leq \frac{\left(p_{0}-p\right)(s-2)}{p-2}$, then

$$
\|u\|_{L^{t p_{2}}} \leq C\|u\|_{L^{p_{0}}} \leq C\|u\|_{V}, v \in V .
$$

Therefore, (H2) follows from (4.12) and (4.15).

(H3) can be verified for $\alpha=p$ in a similar manner.

For $r=\frac{2 p}{d}+p-1$, by the interpolation inequality we have

$$
\left\|f_{0}(u)\right\|_{V^{*}} \leq C\left(1+\|u\|_{L^{r p_{0}^{\prime}}}^{r}\right) \leq C\left(1+\|u\|_{p_{0}}^{p-1}\|u\|_{H}^{\theta}\right), u \in V
$$

where

$$
\frac{1}{p_{0}}+\frac{1}{p_{0}^{\prime}}=1, \quad \theta=\frac{2 p}{d} .
$$

Therefore, (H4) also holds with $\beta=4$.

Then all assertions follow from Theorem 1.2.

Remark 4.5. One further generalization is to replace $\sum_{i=1}^{d} D_{i}\left(\left|D_{i} u\right|^{p-2} D_{i} u\right)$ by more general quasi-linear differential operator

$$
\sum_{|\alpha| \leq m}(-1)^{|\alpha|} D_{\alpha} A_{\alpha}(x, D u(x, t) ; t),
$$

where $D u=\left(D_{\beta} u\right)_{|\beta| \leq m}$. Under certain assumptions (cf. e.g.600, Proposition 30.10]) this operator also satisfies the monotonicity and coercivity conditions. Then by a similar argument, according to Theorem 1.2, we can obtain the existence and uniqueness of solutions to this type of quasi-linear SPDE driven by Lévy noise.

\subsection{Stochastic hydrodynamical systems}

The next example is the stochastic 2D Navier-Stokes equation driven by Lévy noise (cf. [6, 19, 41, 36] for Wiener noise case). The classical Navier-Stokes equation is a very important model in fluid mechanics to describe the time evolution of incompressible fluids, it can be formulated as follows (2D case):

$$
\begin{aligned}
& \partial_{t} u(t)=\nu \Delta u(t)-(u(t) \cdot \nabla) u(t)-\nabla p(t)+f(t), \\
& \nabla \cdot u(t)=0,
\end{aligned}
$$


where $u(t, x)=\left(u^{1}(t, x), u^{2}(t, x)\right)$ represents the velocity field, $\nu$ is the viscosity constant (we keep the standard notation and it should cause no confusion with the measure $\nu$ corresponding to the Lévy process), $p(t, x)$ denotes the pressure and $f$ is an external force field acting on the fluid.

Let $\Lambda$ be a bounded domain in $\mathbb{R}^{2}$ with smooth boundary. Define

$$
V=\left\{v \in W_{0}^{1,2}\left(\Lambda, \mathbb{R}^{2}\right): \nabla \cdot v=0 \text { a.e. in } \Lambda\right\},\|v\|_{V}:=\left(\int_{\Lambda}|\nabla v|^{2} d x\right)^{1 / 2}
$$

and $H$ is the closure of $V$ in the following norm

$$
\|v\|_{H}:=\left(\int_{\Lambda}|v|^{2} d x\right)^{1 / 2}
$$

The linear operator $P_{H}$ (the Helmholtz-Leray projection) and $A$ (Stokes operator with viscosity constant $\nu$ ) are defined by

$$
\begin{gathered}
P_{H}: L^{2}\left(\Lambda, \mathbb{R}^{2}\right) \rightarrow H \quad \text { orthogonal projection; } \\
A: W^{2,2}\left(\Lambda, \mathbb{R}^{2}\right) \cap V \rightarrow H, A u=\nu P_{H} \Delta u .
\end{gathered}
$$

It is well known that the Navier-Stokes equation can be reformulated as follows:

$$
u^{\prime}=A u+F(u)+f_{0}, u(0)=u_{0} \in H,
$$

where $f_{0} \in L^{2}\left(0, T ; V^{*}\right)$ denotes some external force and

$$
F: \mathcal{D}_{F} \subset H \times V \rightarrow H, F(u, v)=-P_{H}[(u \cdot \nabla) v], F(u)=F(u, u) .
$$

It is standard that in the framework of the Gelfand triple

$$
V \subseteq H \equiv H^{*} \subseteq V^{*}
$$

one can show that the following mappings

$$
A: V \rightarrow V^{*}, F: V \times V \rightarrow V^{*}
$$

are well defined. In particular, we have

$$
V^{*}\langle F(u, v), w\rangle_{V}=-_{V^{*}}\langle F(u, w), v\rangle_{V}, V^{*}\langle F(u, v), v\rangle_{V}=0, u, v, w \in V .
$$

Now we consider the stochastic 2D Navier-Stokes equation driven by Lévy noise:

$$
\begin{aligned}
\mathrm{d} X_{t}= & \left(A X_{t}+F\left(X_{t}\right)+f_{0}(t)\right) \mathrm{d} t+B\left(X_{t}\right) \mathrm{d} W_{t} \\
& \quad+\int_{D^{c}} f\left(X_{t-}, z\right) \tilde{N}(\mathrm{~d} t, \mathrm{~d} z)+\int_{D} g\left(X_{t-}, z\right) N(\mathrm{~d} t, \mathrm{~d} z) \\
X_{0}=x &
\end{aligned}
$$


Example 4.6. (Stochastic 2D Navier-Stokes equation) Suppose that $B: V \rightarrow \mathcal{T}_{2}(U ; H)$ and $f, g: \mathbb{R} \times Z \rightarrow \mathbb{R}$ satisfy the following conditions:

$$
\begin{aligned}
& \left\|B\left(v_{1}\right)-B\left(v_{2}\right)\right\|_{2}^{2}+\int_{D^{c}}\left\|f\left(v_{1}, z\right)-f\left(v_{2}, z\right)\right\|_{H}^{2} \nu(\mathrm{d} z) \leq C\left\|v_{1}-v_{2}\right\|_{H}^{2} \\
& \int_{D^{c}}\|f(v, z)\|_{H}^{2} \nu(\mathrm{d} z) \leq C\left(1+\|v\|_{H}^{2}\right) \\
& \int_{D^{c}}\|f(v, z)\|_{H}^{4} \nu(\mathrm{d} z) \leq C\left(1+\|v\|_{H}^{4}\right)
\end{aligned}
$$

where $C$ is some constant.

Then for any $x \in L^{4}\left(\Omega, \mathcal{F}_{0}, \mathbb{P} ; H\right)$, (4.17) has a unique solution $\left\{X_{t}\right\}_{t \in[0, T]}$.

Proof. The hemicontinuity $(H 1)$ is obvious since $A$ is linear and $F$ is bilinear.

Note that $V^{*}\langle F(v), v\rangle_{V}=0$, it is also easy to show that $(H 3)$ holds with $\alpha=2$ :

$$
\begin{gathered}
V^{*}\left\langle A v+F(v)+f_{0}(t), v\right\rangle_{V} \leq-\nu\|v\|_{V}^{2}+\left\|f_{0}(t)\right\|_{V^{*}}\|v\|_{V} \leq-\frac{\nu}{2}\|v\|_{V}^{2}+C\left\|f_{0}(t)\right\|_{V^{*}}^{2}, v \in V, \\
\|B(v)\|_{2}^{2} \leq 2 K\|v\|_{H}^{2}+2\|B(0)\|_{2}^{2}, v \in V .
\end{gathered}
$$

Recall the following estimates (cf. e.g. [41, Lemmas 2.1, 2.2])

$$
\begin{aligned}
& \left.\right|_{V^{*}}\langle F(w), v\rangle_{V} \mid \leq 2\|w\|_{L^{4}\left(\Lambda ; \mathbb{R}^{2}\right)}\|v\|_{V} ; \\
& \left|V^{*}\langle F(w), v\rangle_{V}\right| \leq 2\|w\|_{V}^{3 / 2}\|w\|_{H}^{1 / 2}\|v\|_{L^{4}\left(\Lambda ; \mathbb{R}^{2}\right)}, v, w \in V .
\end{aligned}
$$

Then we have

$$
\begin{aligned}
V^{*}\langle F(u)-F(v), u-v\rangle_{V} & =-_{V^{*}}\langle F(u, u-v), v\rangle_{V}+V^{*}\langle F(v, u-v), v\rangle_{V} \\
& =-V^{*}\langle F(u-v), v\rangle_{V} \\
& \leq 2\|u-v\|_{V}^{3 / 2}\|u-v\|_{H}^{1 / 2}\|v\|_{L^{4}\left(\Lambda ; \mathbb{R}^{2}\right)} \\
& \leq \frac{\nu}{2}\|u-v\|_{V}^{2}+\frac{32}{\nu^{3}}\|v\|_{L^{4}\left(\Lambda ; \mathbb{R}^{2}\right)}^{4}\|u-v\|_{H}^{2}, u, v \in V .
\end{aligned}
$$

Hence we have the local monotonicity:

$$
V^{*}\langle A u+F(u)-A v-F(v), u-v\rangle_{V} \leq-\frac{\nu}{2}\|u-v\|_{V}^{2}+\frac{32}{\nu^{3}}\|v\|_{L^{4}\left(\Lambda ; \mathbb{R}^{2}\right)}^{4}\|u-v\|_{H}^{2} .
$$

Combining with (4.18) we know that (H2) holds with $\rho(v)=C\|v\|_{L^{4}\left(\Lambda ; \mathbb{R}^{2}\right)}^{4}$.

(4.19) and (4.1) imply that (H4) holds with $\beta=2$.

Then it is easy to see that the existence and uniqueness of solutions to (4.17) follows from Theorem 1.2 .

Remark 4.7. As we mentioned in the introduction, besides the stochastic 2D Navier-Stokes equation, many other hydrodynamical systems also satisfy the local monotonicity condition (H2) and coercivity condition (H3). For example, in a recent work of Chueshov and Millet 
[13, they have studied the well-posedness and large deviation principle for an abstract stochastic semilinear equation (driven by Wiener noise) which covers a wide class of fluid dynamical models. In fact, the Condition $(\mathrm{C} 1)$ and $(\mathrm{C} 2)$ in [13] implies that the assumptions in Theorem 1.2 hold. More precisely, (2.2) in [13] implies the coercivity (H3) holds, and the local monotonicity $(H 2)$ follows from (2.4) (or (2.8)) in [13]. Other assumptions in Theorem 1.2 can be also verified easily.

Therefore, Theorem 1.2 can be applied to show the well-posedness of all hydrodynamical models in [13] driven by general Lévy noise instead of Wiener noise, e.g. stochastic magnetohydrodynamic equations, stochastic Boussinesq model for the Bénard convection, stochastic 2D magnetic Bénard problem and stochastic 3D Leray- $\alpha$ model driven by Lévy noise.

\subsection{Stochastic power law fluids}

The next example of SPDE is a model which describes the velocity field of a viscous and incompressible non-Newtonian fluid subject to some random forcing. The deterministic model has been studied intensively in PDE theory (cf. [20, 38] and the references therein). Let $\Lambda$ be a bounded domain in $\mathbb{R}^{d}(d \geq 2)$ with smooth boundary. For a vector field $u: \Lambda \rightarrow \mathbb{R}^{d}$, we denote the rate of strain tensor by

$$
e(u): \Lambda \rightarrow \mathbb{R}^{d} \otimes \mathbb{R}^{d} ; e_{i, j}(u)=\frac{\partial_{i} u_{j}+\partial_{j} u_{i}}{2}, i, j=1, \cdots, d .
$$

Now we consider the case that the extra stress tensor has the following polynomial form:

$$
\tau(u): \Lambda \rightarrow \mathbb{R}^{d} \otimes \mathbb{R}^{d} ; \tau(u)=2 \nu(1+|e(u)|)^{p-2} e(u),
$$

where $\nu>0$ is the kinematic viscosity and $p>1$ is some constant.

In the case of deterministic forcing, the dynamics of power law fluids can be modeled by the following PDE (cf.[38, Chapter 5]):

$$
\begin{aligned}
& \partial_{t} u=\operatorname{div}(\tau(u))-(u \cdot \nabla) u-\nabla p+f, \\
& \operatorname{div}(u)=0,\left.u\right|_{\partial \Lambda}=0, u(0)=u_{0},
\end{aligned}
$$

where $u=u(t, x)=\left(u_{i}(t, x)\right)_{i=1}^{d}$ is the velocity field, $p$ is the pressure, $f$ is some external force and

$$
u \cdot \nabla=\sum_{j=1}^{d} u_{j} \partial_{j}, \quad \operatorname{div}(\tau(u))=\left(\sum_{j=1}^{d} \partial_{j} \tau_{i, j}(u)\right)_{i=1}^{d} .
$$

Remark 4.8. (1) Note that $p=2$ describes the Newtonian fluids and (4.21) reduces to the classical Navier-Stokes equation.

(2) The shear shining fluids (i.e. $p \in(1,2)$ ) and the shear thickening fluids (i.e. $p \in$ $(2, \infty))$ has been also widely studied in different fields of science and engineering (cf. [20, 38]).

Now we consider the following Gelfand triple

$$
V \subset H \subset V^{*},
$$


where

$$
\begin{aligned}
& V=\left\{u \in W_{0}^{1, p}\left(\Lambda ; \mathbb{R}^{d}\right): \nabla \cdot u=0 \text { a.e. in } \Lambda\right\} \\
& H=\left\{u \in L^{2}\left(\Lambda ; \mathbb{R}^{d}\right): \nabla \cdot u=0 \text { a.e. in } \Lambda, u \cdot n=0 \text { on } \partial \Lambda\right\} .
\end{aligned}
$$

Let $P_{H}$ be the orthogonal (Helmhotz-Leray) projection from $L^{2}\left(\Lambda, \mathbb{R}^{d}\right)$ to $H$. Similarly as in the previous example, we can show that the following operators

$$
\begin{gathered}
A: W^{2, p}\left(\Lambda ; \mathbb{R}^{d}\right) \cap V \rightarrow H, A(u):=P_{H}[\operatorname{div}(\tau(u))] \\
F: W^{2, p}\left(\Lambda ; \mathbb{R}^{d}\right) \cap V \times W^{2, p}\left(\Lambda ; \mathbb{R}^{d}\right) \cap V \rightarrow H ; F(u, v):=-P_{H}[(u \cdot \nabla) v], F(u):=F(u, u)
\end{gathered}
$$

can be extended to the well defined operators:

$$
A: V \rightarrow V^{*} ; F: V \times V \rightarrow V^{*}
$$

In particular, one can show that

$$
\begin{gathered}
V^{*}\langle A(u), v\rangle_{V}=-\int_{\Lambda} \sum_{i, j=1}^{d} \tau_{i, j}(u) e_{i, j}(v) \mathrm{d} x, u, v \in V \\
V^{*}\langle F(u, v), w\rangle_{V}=-V^{*}\langle F(u, w), v\rangle_{V}, V^{*}\langle F(u, v), v\rangle_{V}=0, u, v, w \in V .
\end{gathered}
$$

Now we consider stochastic equation of power law fluids driven by Lévy noise:

$$
\begin{aligned}
\mathrm{d} X_{t}= & \left(A X_{t}+F\left(X_{t}\right)+f_{0}(t)\right) \mathrm{d} t+B\left(X_{t}\right) \mathrm{d} W_{t} \\
& \quad+\int_{D^{c}} f\left(X_{t-}, z\right) \tilde{N}(\mathrm{~d} t, \mathrm{~d} z)+\int_{D} g\left(X_{t-}, z\right) N(\mathrm{~d} t, \mathrm{~d} z) \\
X_{0}=x &
\end{aligned}
$$

where $f_{0}:=P_{H} f$.

Example 4.9. (Stochastic equation of power law fluids) Suppose that $f_{0} \in L^{2}([0, T] ; H)$, $B: V \rightarrow \mathcal{T}_{2}(U ; H)$ and $f, g: \mathbb{R} \times Z \rightarrow \mathbb{R}$ satisfy the following conditions:

$$
\begin{aligned}
& \left\|B\left(v_{1}\right)-B\left(v_{2}\right)\right\|_{2}^{2}+\int_{D^{c}}\left\|f\left(v_{1}, z\right)-f\left(v_{2}, z\right)\right\|_{H}^{2} \nu(\mathrm{d} z) \leq C\left\|v_{1}-v_{2}\right\|_{H}^{2} \\
& \int_{D^{c}}\|f(v, z)\|_{H}^{2} \nu(\mathrm{d} z) \leq C\left(1+\|v\|_{H}^{2}\right) \\
& \int_{D^{c}}\|f(v, z)\|_{H}^{4} \nu(\mathrm{d} z) \leq C\left(1+\|v\|_{H}^{4}\right)
\end{aligned}
$$

where $C$ is some constant.

Then if $p \geq \frac{d+2}{2}$, for any $x \in L^{4}\left(\Omega, \mathcal{F}_{0}, \mathbb{P} ; H\right)$ (4.22) has a unique solution $\left\{X_{t}\right\}_{t \in[0, T]}$. 
Proof. Without loss of generality we may assume the viscosity constant $\nu=1$.

We first recall the well known Korn's inequality for $p \in(1, \infty)$ (cf. [38, Theorem 1.10 (pp.196)]):

$$
\int_{\Lambda}|e(u)|^{p} \mathrm{~d} x \geq C_{p}\|u\|_{1, p}, u \in W_{0}^{1, p}\left(\Lambda ; \mathbb{R}^{d}\right),
$$

where $C_{p}>0$ is some constant.

The following inequalities are also used very often in the study of power law fluids (cf. [38, pp.198 Lemma 1.19]):

$$
\begin{aligned}
& \left|\tau_{i, j}(u)\right| \leq C(1+|e(u)|)^{p-1}, i, j=1, \cdots, d ; \\
& \sum_{i, j=1}^{d} \tau_{i, j}(u) e_{i, j}(u) \geq C\left(|e(u)|^{p}-1\right) ; \\
& \sum_{i, j=1}^{d}\left(\tau_{i, j}(u)-\tau_{i, j}(v)\right)\left(e_{i, j}(u)-e_{i, j}(v)\right) \geq C\left(|e(u)-e(v)|^{2}+|e(u)-e(v)|^{p}\right) .
\end{aligned}
$$

Then by the interpolation inequality and Young's inequality one can show that

$$
\begin{aligned}
& V^{*}\langle F(u)-F(v), u-v\rangle_{V} \\
& =-V^{*}\langle F(u-v), v\rangle_{V} \\
& =V^{*}\langle F(u-v, v), u-v\rangle_{V} \\
& \leq C\|v\|_{V}\|u-v\|_{L^{\frac{2 p}{p-1}}}^{2} \\
& \leq C\|v\|_{V}\|u-v\|_{1,2}^{\frac{d}{p}}\|u-v\|_{H}^{\frac{2 p-d}{p}} \\
& \leq \varepsilon\|u-v\|_{1,2}^{2}+C_{\varepsilon}\|v\|_{V}^{\frac{2 p}{2 p-d}}\|u-v\|_{H}^{2}, u, v \in V .
\end{aligned}
$$

By (4.24) and Korn's inequality we have

$$
\begin{aligned}
& V^{*}\langle A(u)-A(v), u-v\rangle_{V} \\
= & -\int_{\Lambda} \sum_{i, j=1}^{d}\left(\tau_{i, j}(u)-\tau_{i, j}(v)\right)\left(e_{i, j}(u)-e_{i, j}(v)\right) \mathrm{d} x \\
\leq & -C\|e(u)-e(v)\|_{H}^{2} \\
\leq & -C\|u-v\|_{1,2}^{2} .
\end{aligned}
$$

Hence we have the following estimate:

$$
V^{*}\langle A(u)+F(u)-A(v)-F(v), u-v\rangle_{V} \leq-(C-\varepsilon)\|u-v\|_{1,2}^{2}+C_{\varepsilon}\|v\|_{V}^{\frac{2 p}{2 p-d}}\|u-v\|_{H}^{2},
$$

where $\varepsilon>0$ and $C_{\varepsilon}$ are some constants.

Hence (H2) holds with $\rho(v)=C_{\varepsilon}\|v\|_{V}^{\frac{2 p}{2 p-d}}$. 
It is also easy to verify (H3) with $\alpha=p$ as follows:

$$
V^{*}\langle A(v)+F(v), v\rangle_{V} \leq-C_{1} \int_{\Lambda}|e(v)|^{p} \mathrm{~d} x+C_{2} \leq-C_{3}\|v\|_{V}^{p}+C_{2},
$$

where $C_{1}, C_{2}, C_{3}$ are some constants.

Note that

$$
\left|V_{V^{*}}\langle F(v), u\rangle_{V}\right|=\left.\right|_{V^{*}}\langle F(v, u), v\rangle_{V} \mid \leq\|u\|_{V}\|v\|_{L^{\frac{2 p}{p-1}}}^{2}, u, v \in V,
$$

hence we have

$$
\|F(v)\|_{V^{*}} \leq\|v\|_{L^{\frac{2 p}{p-1}}}^{2}, v \in V .
$$

Then by the interpolation inequality and Sobolev's inequality we have

$$
\|v\|_{L^{\frac{2 p}{p-1}}} \leq\|v\|_{L^{q}}^{\gamma}\|v\|_{L^{2}}^{1-\gamma} \leq C\|v\|_{V}^{\gamma}\|v\|_{H}^{1-\gamma}
$$

where $q=\frac{d p}{d-p}$ and $\gamma=\frac{d}{(d+2) p-2 d}$.

Note that $2 \gamma \leq p-1$ if $p \geq \frac{2+d}{2}$, and it is also easy to see that

$$
\|A(v)\|_{V^{*}} \leq C\left(1+\|v\|_{V}^{p-1}\right), v \in V .
$$

Hence the growth condition (H4) also holds.

Moreover, note that $d \geq \frac{2+d}{2}$, it is easy to show that (1.4) also holds. 1.2 .

Therefore, the existence and uniqueness of solutions to (4.22) follows from Theorem

Remark 4.10. In [57] the authors established the existence and uniqueness of weak solutions for (4.22) with additive Wiener noise. They first considered the Galerkin approximation and showed the tightness of the distributions of the corresponding approximating solutions. Then they proved that the limit is a weak solution of (4.22) with additive Wiener noise.

In 37] the authors obtained the existence and uniqueness of strong solutions for (4.22) with multiplicative Wiener noise. Here by applying Theorem 1.2 we establish the existence and uniqueness of strong solutions to (4.22) in $L^{2}$-space of divergence free vector fields with multiplicative Lévy noise.

\section{References}

[1] S. Albeverio, Z. Brzeźniak and J.-L. Wu, Existence of global solutions and invariant measures for stochastic differential equations driven by Poisson type noise with nonLipschitz coefficients, J. Math. Anal. Appl. 371 (2010), 309-322.

[2] S. Albeverio, J. Wu, and T. Zhang. Parabolic SPDEs driven by Poisson white noise, Stoc. Proc. Appl. 74 (1998), 21-36. 
[3] D. Applebaum, J.-L.Wu, Stochastic partial differential equations driven by Lévy space time white noise, Random Oper. Stochastic Equations 8 (2000), 245-259.

[4] G. A. Anastassiou and S. S. Dragomir, On some estimates of the remainder in Taylor's formula, J. Math. Anal. Appl. 263 (2001), no. 1, 246-263.

[5] L. Bo, K. Shi and Y. Wang Variational solutions of dissipative jump-type stochastic evolution equations, J. Math. Anal. Appl. 373 (2011), 111-126.

[6] Z. Brzeźniak, M. Capiński and F. Flandoli, Stochastic Navier-Stokes Equations with Multiplicative Noise, Stoc. Anal. Appl. 105 (1992), 523-532.

[7] Z. Brzeźniak, B. Goldys and M. Neklyudov, Multidimensional stochastic Burgers equation, arXiv:1202.3230.

[8] Z. Brzeźniak, E. Hausenblas, Maximal regularity for stochastic convolutions driven by Lévy processes, Probab. Theory Related Fields 145 (2009), no. 3-4, 615-637.

[9] Z. Brzeźniak, E. Hausenblas, Martingale solutions for Stochastic Equation of Reaction Diffusion Type driven by Lévy noise or Poisson random measure, arXiv:1010.5933.

[10] Z. Brzeźniak, E. Hausenblas and J. Zhu, 2D stochastic Navier-Stokes equations driven by jump noises, Nonlinear Anal. 79 (2013), 122-139.

[11] Z. Brzeźniak and J. Zabczyk, Regularity of Ornstein-Uhlenbeck processes driven by a Lévy white noise, Potential Anal. 32 (2010), 153-188.

[12] Z. Brzeźniak and J. Zhu, Stochastic beam equations driven by compensated Poisson random measures, arXiv:1011.5377.

[13] I. Chueshov and A. Millet, Stochastic 2D hydrodynamical type systems: well posedness and large deviations, Appl. Math. Optim. 61 (2010), 379-420.

[14] G. Deugoue and M. Sango, On the Strong Solution for the 3D Stochastic Leray-Alpha Model, Boundary Value Problems vol. 2010, 1-32.

[15] N. Dinculeanu, Vector integration and stochastic integration in Banach spaces, Pure and Applied Mathematics (New York). Wiley-Interscience, New York, 2000.

[16] Z. Dong and T.G. Xu, One-dimensional stochastic Burgers equation driven by Lévy processes, J. Funct. Anal. 243 (2007), 631-678.

[17] Z. Dong and Y. Xie, Global solutions of stochastic 2D Navier-Stokes equations with Lèvy noise, Sci. China Ser. A 52 (2009), no. 7, 1497-1524.

[18] Z. Dong, L. Xu and X. Zhang, Invariance measures of stochastic 2D Navier-Stokes equations driven by $\alpha$-stable processes, Electron. Commun. Probab. 16 (2011), 678-688. 
[19] F. Flandoli, D. Ga̧tarek, Martingale and stationary solutions for stochastic NavierStokes equations, Probab. Theory Related Fields 102 no. 3 (1995), 367-391

[20] J. Frehse and M. Rưžička, Non-homogeneous generalized Newtonian fluids, Math. Z. 260 (2008), 355-375.

[21] B. Gess, W. Liu and M. Röckner, Random attractors for a class of stochastic partial differential equations driven by general additive noise, J. Differential Equations 251 (2011), 1225-1253.

[22] I. Gyöngy and N. V. Krylov, On stochastic equations with respect to semimartingales. I. , Stochastics 4 (1980), 1-21.

[23] I. Gyöngy and N. V. Krylov, On stochastics equations with respect to semimartingales. II. Itô formula in Banach spaces, Stochastics 6 (1982), 153-173.

[24] I. Gyöngy, On stochastic equations with respect to semimartingale III, Stochastics 7 (1982), 231-254.

[25] E. Hausenblas, Existence, Uniqueness and Regularity of Parabolic SPDEs driven by Poisson random measure, Electron. J. Probab. 10 (2005), 1496-1546.

[26] E. Hausenblas, SPDEs driven by Poisson random measures with non Lipschitz coefficients: existence results, Probab. Theory Related Fields 137 (2007), no. 1-2, 161-200.

[27] G. Kallianpur, J. Xiong, Stochastic differential equations in infinite dimensional spaces, Lecture Notes - Monograph Series, vol. 26. Institute of Mathematical Statistics, 1996

[28] A. N. Kolmogorov and S. V. Fomin, Elementi di teoria delle funzioni e di analisi funzionale, Edizione MIR, Moscau, Translation from the russian: Elementy teorii funktsij i funktisianal'nogo, Copyright by NAUKA, Moscau, 1980.

[29] N.V. Krylov and B.L. Rozovskii, Stochastic evolution equations, Translated from Itogi Naukii Tekhniki, Seriya Sovremennye Problemy Matematiki 14 (1979), 71-146.

[30] A. Ichikawa, Some inequalities for martingales and stochastic convolutions, Stochastic Anal. Appl. 4 (1986),, no. 3, 329-339.

[31] N. Ikeda, S. Watanabe, Stochastic Differential Equations and Diffusion Processes, North-Holland and Kodansha, Tokyo, 1981.

[32] K. Itô, On stochastic processes, Japan J. Math. 18 (1942), 261-301.

[33] K. Itô, Poisson point processes attached to Markov processes, Proceedings of the Sixth Berkeley Symposium on Mathematical Statistics and Probability (Univ. California, Berkeley, Calif., 1970/1971), Vol. III: Probability theory, pp. 225-239. Univ. California Press, Berkeley, California, 1972. 
[34] W. Liu, Large deviations for stochastic evolution equations with small multiplicative noise, Appl. Math. Optim. 61 (2010), 27-56.

[35] W. Liu, Existence and Uniqueness of Solutions to Nonlinear Evolution Equations with Locally Monotone Operators, Nonlinear Anal. 74 (2011), 7543-7561.

[36] W. Liu and M. Röckner, SPDE in Hilbert space with locally monotone coefficients, J. Funct. Anal. 259 (2010), 2902-2922.

[37] W. Liu and M. Röckner, Local and global well-posedness of SPDE with generalized coercivity conditions, J. Differential Equations 254 (2013), 725-755.

[38] J. Málek, J. Nečas, M. Rokyta and M. Rưžička, Weak and measure-valued solutions to evolutionary PDEs, Applied Mathematics and Mathematical Computation, vol. 13, Chapman \& Hall, London, 1996.

[39] V. Mandrekar and B. Rüdiger, Existence and uniqueness of path wise solutions for stochastic integral equations driven by Lévy noise on separable Banach spaces, Stochastics 78 (2006), no. 4, 189-212.

[40] C. Marinelli and M. Röckner, Well-posedness and asymptotic behavior for stochastic reaction-diffusion equations with multiplicative poisson noise, Electron. J. Probab. 49 (2010), 1528-1555.

[41] J.-L. Menaldi and S.S. Sritharan, Stochastic 2D Navier-Stokes equation, Appl. Math. Optim. 46 (2002), 31-53.

[42] M. Métivier, Semimartingales. A course on stochastic processes, de Gruyter Studies in Mathematics, 2. Walter de Gruyter Co., Berlin-New York, 1982.

[43] C. Mueller, The heat equation with Lévy noise, Stochastic Process. Appl. 74 (1998), 67-82.

[44] C. Mueller, L. Mytnik, and A. Stan, The heat equation with time-independent multiplicaive stable Lévy noise, Stochastic Process. Appl. 116 (2006), 70-100.

[45] L. Mytnik, Stochastic partial differential equation driven by stable noise, Probab. Theory Related Fields, 123 (2002), 157-201.

[46] E. Pardoux, Equations aux dérivées partielles stochastiques non linéaires monotones, Ph.D. thesis, Université Paris XI, 1975.

[47] S. Peszat and J. Zabczyk, Stochastic partial differential equations with Lévy noise: An evolution equation approach, Encyclopedia of Mathematics and its Applications, vol. 113, Cambridge University Press, Cambridge, 2007.

[48] C. Prévôt and M. Röckner, A concise course on stochastic partial differential equations, Lecture Notes in Mathematics, vol. 1905, Springer, 2007. 
[49] J. Ren, M. Röckner and F.-Y. Wang, Stochastic generalized porous media and fast diffusion equations, J. Differential Equations 238 (2007), 118-152.

[50] M. Röckner, Introduction to Stochastic Partial Differential Equations, Lecture Notes, 2013.

[51] M. Röckner and F.-Y. Wang, Non-monotone stochastic porous media equation, J. Differential Equations 245 (2008), 3898-3935.

[52] M. Röckner and T. Zhang, Stochastic evolution equations of jump type: existence, uniqueness and large deviation principles, Potential Anal. 26 (2007), 255-279.

[53] B. Rüdiger, Stochastic integration with respect to compensated Poisson random measures on separable Banach spaces, Stoch. Stoch. Rep. 76 (2004), no. 3, 213-242.

[54] B. Schmalfuss, Qualitative properties for the stochastic Navier-Stokes equation, Nonlinear Anal. 28 (1997), no. 9, 1545-1563.

[55] S.S. Sritharan and P. Sundar, Large deviations for the two-dimensional Navier-Stokes equations with multiplicative noise, Stochastic Process. Appl. 116 (2006), 1636-1659.

[56] R. Temam, Navier-Stokes Equations, Reprint of the 1984 edition. AMS Chelsea Publishing, Providence, RI, 2001

[57] Y. Terasawa and N. Yoshida, Stochastic power law fluids: Existence and uniqueness of weak solutions, Ann. Appl. Probab. 21 (2011) , 1827-1859.

[58] A. Truman, J.-L. Wu, Stochastic Burgers equation with Lévy space-time white noise, Probabilistic methods in fluids, 298-323, World Sci. Publ., River Edge, NJ, 2003.

[59] X. Zhang, On stochastic evolution equations with non-Lipschitz coefficients, Stoc. Dyna. 9 (2009), 549-595.

[60] E. Zeidler, Nonlinear Functional Analysis and its Applications II/B: Nonlinear Monotone operators, Springer-Verlag, New York, 1990.

[61] J. Zhu, A Study of SPDEs w.r.t. Compensated Poisson Random Measures and Related Topics, Ph. D. Thesis. The University of York, 2010. 